\title{
The structure of the perturbation series of the spin-1 Bose gas at low temperatures
}

\author{
Péter Szépfalusy ${ }^{(1,2)}$ and Gergely Szirmai ${ }^{(1)}$ \\ (1) Department of Physics of Complex Systems, Roland Eötvös University, Pázmány Péter sétány 1/A, Budapest, H-111\%, \\ ${ }^{(2)}$ Research Institute for Solid State Physics and Optics of the Hungarian Academy of Sciences, Budapest, P.O.Box 49, H-1525
}

(November 5, 2018)

\begin{abstract}
The properties of Green's functions and various correlation functions of density and spin operators are considered in a homogeneous spin-1 Bose gas in different phases. The dielectric formalism is worked out and the partial coincidence of the one-particle and collective spectra is pointed out below the temperature of Bose-Einstein condensation. As an application the formalism is used to give two approximations for the propagators and the correlation functions and the spectra of excitations including shifts and widths due to the thermal cloud.
\end{abstract}

PACS numbers: 03.75.Fi, 67.40.Db, 05.30.Jp

\section{INTRODUCTION}

The recent realization of BEC in an optical trap has opened a new area of research [1 15 . The theoretical investigation of spinor systems is growing rapidly these days concerning the ground state structure and symmetry braking [6 9., the various phases in traps [10,11], the different vortex states [12, 13], the exploration of collective excitations 114 17] and a number of other problems [18 27]. Compared to the BEC realized in magnetic traps [28,29], where the spin degree of freedom of the particle field is frozen, such systems, since the spinor nature of the particles is preserved, have a wider variety of excitations including spin density waves, transverse spin waves or quadrupolar spin waves.

It is a well established property of scalar Bose-Einstein condensed systems that the one-particle and the density correlation function spectra coincide. To treat this problem consistently the dielectric formalism has proved to be particularly useful, which has been worked out first for homogeneous systems 30 35] and recently generalized and applied to trap systems [36 39]. In this paper this formalism is extended to gases with a spinor Bose-Einstein condensate. To make the presentation more transparent only homogeneous systems will be considered. The new feature is the appearance of correlation functions including spin fluctuations besides the density autocorrelation function and a variety of new one-particle Green's functions. Their perturbation series are analysed simultaneously. After suitable rearrangement of the expansions it has been achieved that certain one-particle Green's functions and correlation functions have common denominators leading to the coincidence of their spectra, though with different spectral weights. Examples are the Green's functions corresponding to a spin transfer zero and the density correlation function, furthermore those Green's functions and correlation functions of the spin operators, which can be characterized by spin transfers +1 or -1 . Exceptions are found in the polar phase (occurring when the interaction in the spin channel is repulsive), namely those correlation functions of the spin operators which create zero or \pm 2 spin transfers do not possess condensate induced intermixing with the one-particle Green's functions. The general theory is illustrated in the Bogoliubov theory (valid at very low temperatures) and in the random phase approximation (RPA). Within the RPA we do not include exchange processes, that already in the case of the scalar Bose-Einstein condensed systems has led to involved calculations [39]. Their extension to spinor Bose-Einstein gas will be presented in a separate paper. As a matter of fact the relative importance of the exchange processes is less in the present case since the direct terms are enlarged by summations over spin variables.

Though the RPA (as a mean field theory) looses its validity near the phase transition it is enlightening to investigate it in the transition region. It turns out that, while the transition to the polar phase is continuous when decreasing the temperature, it becomes weakly first order for ferromagnetic ordering due to the small (for this coupling attractive) spin dependent part of the interaction. It is worth recalling that in case of the scalar Bose-Einstein condensation the transition is second order in this RPA-Hartree model, but including exchange contributions makes it to a first order one and the effect is then not small, see Ref. [39] and references therein.

The paper is organized as follows. In the second section, after giving the specifications of the Hamiltonian and introducing the canonical transformation to induce the Bose-Einstein condensation, the normal and anomalous oneparticle Green's functions (matrices in the spin variables) and the different correlation functions of the particle number density and spin density operators are defined. Section III is the backbone of the paper. It starts with a summary of symmetry properties of the functions introduced in Section II. Then their general structure is analyzed within the framework of perturbation expansion. We proceed by classifying the processes according to the spin transfers involved. Then in the spirit of the dielectric formalism the proper irreducible graphs are separated. The main results 
are the description of condensate induced intermixing of one-particle and collective modes and the specification of the conditions when it occurs. Sections $[\mathrm{V}$ and $\mathrm{V}$ are devoted to approximate calculations. In Section $\mathrm{V}$ corrections consisting of damping terms and frequency shifts as well are given in RPA to the Bogoliubov approximation discussed in Section IV. We present in Section $\square$ also results for static properties in RPA. Section VI contains the summary and further discussion.

\section{BASIC EQUATIONS AND DEFINITIONS}

\section{A. The effective Hamiltonian of the spin-1 Bose gas}

In a gas containing spin-1 particles the wavefunction is a three-component spinor. To be more specific we use the basis set of $\widehat{F}_{z}$ eigenvectors in the spin space, which leads to the wavefunction

$$
\psi(\mathbf{r})=\left(\begin{array}{c}
\psi_{+}(\mathbf{r}) \\
\psi_{0}(\mathbf{r}) \\
\psi_{-}(\mathbf{r})
\end{array}\right)
$$

In this representation the spin operators are

$$
F_{x}=\frac{1}{\sqrt{2}}\left[\begin{array}{lll}
0 & 1 & 0 \\
1 & 0 & 1 \\
0 & 1 & 0
\end{array}\right], \quad F_{y}=\frac{1}{\sqrt{2}}\left[\begin{array}{ccc}
0 & -i & 0 \\
i & 0 & -i \\
0 & i & 0
\end{array}\right], \quad F_{z}=\left[\begin{array}{ccc}
1 & 0 & 0 \\
0 & 0 & 0 \\
0 & 0 & -1
\end{array}\right],
$$

and the corresponding raising and lowering operators:

$$
F_{+}=\sqrt{2}\left[\begin{array}{lll}
0 & 1 & 0 \\
0 & 0 & 1 \\
0 & 0 & 0
\end{array}\right], \quad F_{-}=\sqrt{2}\left[\begin{array}{lll}
0 & 0 & 0 \\
1 & 0 & 0 \\
0 & 1 & 0
\end{array}\right]
$$

We consider a system of spin-1 particles in a box with periodic boundary conditions and without an external potential. The interaction between the particles is an s-wave scattering. Such a rotationally invariant scattering interaction can be described by a potential

$$
V\left(\mathbf{r}_{1}-\mathbf{r}_{2}\right)=\delta\left(\mathbf{r}_{1}-\mathbf{r}_{2}\right)\left[c_{n}+c_{s} \mathbf{F}_{1} \cdot \mathbf{F}_{2}\right]
$$

where the constants $c_{n}$ and $c_{s}$ is connected to the s-wave scattering lengths $\left(g_{0}\right.$ and $\left.g_{2}\right)$ in the total hyperfine spin channel zero and two with $c_{n}=\left(g_{0}+2 g_{2}\right) / 3$ and $c_{s}=\left(g_{2}-g_{0}\right) / 3[14]$.

In the second quantized formalism we introduce annihilation and creation operators $a_{r}(\mathbf{k})$ and $a_{r}^{\dagger}(\mathbf{k})$ which destroy and create one particle states of plane waves with momentum $\mathbf{k}$ and spin projection $r$. These operators are bosonic in our case, so they satisfy the commutation relations:

$$
\begin{array}{r}
{\left[a_{r}(\mathbf{k}), a_{s}^{\dagger}\left(\mathbf{k}^{\prime}\right)\right]=\delta_{r, s} \delta\left(\mathbf{k}-\mathbf{k}^{\prime}\right),} \\
{\left[a_{r}(\mathbf{k}), a_{s}\left(\mathbf{k}^{\prime}\right)\right]=\left[a_{r}^{\dagger}(\mathbf{k}), a_{s}^{\dagger}\left(\mathbf{k}^{\prime}\right)\right]=0 .}
\end{array}
$$

In this formalism the grand canonical Hamiltonian of the system reads as:

$$
\mathcal{H}=\sum_{\mathbf{k}}\left(e_{\mathbf{k}}-\mu\right) a_{r}^{\dagger}(\mathbf{k}) a_{r}(\mathbf{k})+\frac{1}{2} \sum_{\substack{\mathbf{k}_{1}+\mathbf{k}_{2}=\\=\mathbf{k}_{3}+\mathbf{k}_{4}}} a_{r^{\prime}}^{\dagger}\left(\mathbf{k}_{1}\right) a_{r}^{\dagger}\left(\mathbf{k}_{2}\right) V_{r s}^{r^{\prime} s^{\prime}} a_{s}\left(\mathbf{k}_{3}\right) a_{s^{\prime}}\left(\mathbf{k}_{4}\right),
$$

where $e_{\mathbf{k}}=\hbar^{2} k^{2} / 2 M$ stands for the kinetic energy of a particle and $\mu$ is the chemical potential of the system and

$$
V_{r s}^{r^{\prime} s^{\prime}}=c_{n} \delta_{r s} \delta_{r^{\prime} s^{\prime}}+c_{s}(\mathbf{F})_{r s}(\mathbf{F})_{r^{\prime} s^{\prime}}
$$

according to (4). Here and in the following the convention of summing over repeated indices is applied except when stated otherwise.

There are two types of systems depending on the sign of $c_{s}$. If it is less than zero then the spins prefer parallel alignment which leads to a macroscopic magnetization in the presence of a Bose-Einstein condensate. If $c_{s}$ is greater than zero then the energetically favorable state is when $\langle\mathbf{F}\rangle=0$. The former case is called ferromagnetic case and later is called polar case [14]. 


\section{B. Description of the symmetry breaking}

In the Bose-Einstein condensed phases some field operators have anomalous averages reflecting a broken gauge symmetry: $\left\langle a_{r}(0)\right\rangle=\sqrt{N_{0}} \zeta_{r}$ and $\left\langle a_{r}^{\dagger}(0)\right\rangle=\sqrt{N_{0}} \zeta_{r}^{\dagger}$ where $N_{0}$ is the number of particles in the condensate and $\zeta_{r}$ is the normalized spinor of the condensate 14]. For the polar case one can take $\zeta_{r}$ as $(0,1,0)^{T}$ and for the ferromagnetic case $\zeta_{r}$ can be taken as $(1,0,0)^{T}$ where the superscript $T$ denotes the operation of transposition. The averaging is made over a symmetry breaking ensemble. To consider this symmetry breaking, one can introduce a new set of bosonic annihilation and creation operators with a canonical transformation:

$$
\begin{aligned}
& b_{r}(\mathbf{k})=a_{r}(\mathbf{k})-\delta_{\mathbf{k}, 0} \sqrt{N_{0}} \zeta_{r}, \\
& b_{r}^{\dagger}(\mathbf{k})=a_{r}^{\dagger}(\mathbf{k})-\delta_{\mathbf{k}, 0} \sqrt{N_{0}} \zeta_{r}^{\dagger} .
\end{aligned}
$$

The relation between the chemical potential and condensate density can be derived from the requirement that $\left\langle b_{r}(\mathbf{k})\right\rangle=$ $\left\langle b_{r}^{\dagger}(\mathbf{k})\right\rangle=0$ Here and from now on the averages will be made over the grand canonical ensemble with a density matrix $\rho=\mathcal{Z}^{-1} e^{\beta \mathcal{H}}$ where $\mathcal{Z}=\operatorname{Tr} \mathrm{e}^{\beta \mathcal{H}}$ is the grand canonical partition function. Substituting the canonical transformation into (6) one obtains the Hamiltonian in terms of the new operators:

$$
\begin{aligned}
& \mathcal{H}=\sum_{\mathbf{k}}\left(e_{\mathbf{k}}-\mu_{0}\right) b_{r}^{\dagger}(\mathbf{k}) b_{r}(\mathbf{k})-\mu \sqrt{N_{0}}\left[\zeta_{r}^{\dagger} b_{r}(0)+b_{r}^{\dagger}(0) \zeta_{r}\right]-\mu N_{0}+\frac{1}{2} \sum b_{r^{\prime}}^{\dagger}\left(\mathbf{k}_{1}\right) b_{r}^{\dagger}\left(\mathbf{k}_{2}\right) V_{r s}^{r^{\prime} s^{\prime}} b_{s}\left(\mathbf{k}_{3}\right) b_{s^{\prime}}\left(\mathbf{k}_{4}\right) \\
& +\frac{\sqrt{N_{0}}}{2} \sum b_{r^{\prime}}^{\dagger}\left(\mathbf{k}_{1}\right) b_{r}^{\dagger}\left(\mathbf{k}_{2}\right) V_{r s}^{r^{\prime} s^{\prime}} b_{s}\left(\mathbf{k}_{3}\right) \zeta_{s^{\prime}} \delta_{\mathbf{k}_{4}, 0}+\frac{\sqrt{N_{0}}}{2} \sum b_{r^{\prime}}^{\dagger}\left(\mathbf{k}_{1}\right) b_{r}^{\dagger}\left(\mathbf{k}_{2}\right) V_{r s}^{r^{\prime} s^{\prime}} \zeta_{s} \delta_{\mathbf{k}_{3}, 0} b_{s^{\prime}}\left(\mathbf{k}_{4}\right) \\
& +\frac{\sqrt{N_{0}}}{2} \sum b_{r^{\prime}}^{\dagger}\left(\mathbf{k}_{1}\right) \zeta_{r}^{\dagger} \delta_{\mathbf{k}_{2}, 0} V_{r s}^{r^{\prime} s^{\prime}} b_{s}\left(\mathbf{k}_{3}\right) b_{s^{\prime}}\left(\mathbf{k}_{4}\right)+\frac{\sqrt{N_{0}}}{2} \sum \zeta_{r^{\prime}}^{\dagger} \delta_{\mathbf{k}_{1}, 0} b_{r}^{\dagger}\left(\mathbf{k}_{2}\right) V_{r s}^{r^{\prime} s^{\prime}} b_{s}\left(\mathbf{k}_{3}\right) b_{s^{\prime}}\left(\mathbf{k}_{4}\right) \\
& +\frac{N_{0}}{2} \sum b_{r^{\prime}}^{\dagger}\left(\mathbf{k}_{1}\right) b_{r}^{\dagger}\left(\mathbf{k}_{2}\right) V_{r s}^{r^{\prime} s^{\prime}} \zeta_{s} \delta_{\mathbf{k}_{3}, 0} \zeta_{s^{\prime}} \delta_{\mathbf{k}_{4}, 0}+\frac{N_{0}}{2} \sum b_{r^{\prime}}^{\dagger}\left(\mathbf{k}_{1}\right) \zeta_{r}^{\dagger} \delta_{\mathbf{k}_{2}, 0} V_{r s}^{r^{\prime} s^{\prime}} b_{s}\left(\mathbf{k}_{3}\right) \zeta_{s^{\prime}} \delta_{\mathbf{k}_{4}, 0} \\
& +\frac{N_{0}}{2} \sum \zeta_{r^{\prime}}^{\dagger} \delta_{\mathbf{k}_{1}, 0} b_{s}^{\dagger}\left(\mathbf{k}_{2}\right) V_{r s}^{r^{\prime} s^{\prime}} b_{s}\left(\mathbf{k}_{3}\right) \zeta_{s^{\prime}} \delta_{\mathbf{k}_{4}, 0}+\frac{N_{0}}{2} \sum b_{r^{\prime}}^{\dagger}\left(\mathbf{k}_{1}\right) \zeta_{r}^{\dagger} \delta_{\mathbf{k}_{2}, 0} V_{r s}^{r^{\prime} s^{\prime}} \zeta_{s} \delta_{\mathbf{k}_{3}, 0} b_{s^{\prime}}\left(\mathbf{k}_{4}\right) \\
& +\frac{N_{0}}{2} \sum \zeta_{r^{\prime}}^{\dagger} \delta_{\mathbf{k}_{1}, 0} b_{r}^{\dagger}\left(\mathbf{k}_{2}\right) V_{r s}^{r^{\prime} s^{\prime}} \zeta_{s} \delta_{\mathbf{k}_{3}, 0} b_{s^{\prime}}\left(\mathbf{k}_{4}\right)+\frac{N_{0}}{2} \sum \zeta_{r^{\prime}}^{\dagger} \delta_{\mathbf{k}_{1}, 0} \zeta_{r}^{\dagger} \delta_{\mathbf{k}_{2}, 0} V_{r s}^{r^{\prime} s^{\prime}} b_{s}\left(\mathbf{k}_{3}\right) b_{s^{\prime}}\left(\mathbf{k}_{4}\right) \\
& +\frac{N_{0}^{3 / 2}}{2} \zeta_{r^{\prime}}^{\dagger} \zeta_{r}^{\dagger} V_{r s}^{r^{\prime} s^{\prime}} \zeta_{s} b_{s^{\prime}}(0)+\frac{N_{0}^{3 / 2}}{2} \zeta_{r^{\prime}}^{\dagger} \zeta_{r}^{\dagger} V_{r s}^{r^{\prime} s^{\prime}} b_{s}(0) \zeta_{s^{\prime}}+\frac{N_{0}^{3 / 2}}{2} \zeta_{r^{\prime}}^{\dagger} b_{r}^{\dagger}(0) V_{r s}^{r^{\prime} s^{\prime}} \zeta_{s} \zeta_{s^{\prime}} \\
& +\frac{N_{0}^{3 / 2}}{2} b_{r^{\prime}}^{\dagger}(0) \zeta_{r}^{\dagger} V_{r s}^{r^{\prime} s^{\prime}} \zeta_{s} \zeta_{s^{\prime}}+\frac{N_{0}^{2}}{2} \zeta_{r^{\prime}}^{\dagger} \zeta_{r}^{\dagger} V_{r s}^{r^{\prime} s^{\prime}} \zeta_{s} \zeta_{s^{\prime}}+\sum_{\mathbf{k}}\left(\mu_{0}-\mu\right) b_{r}^{\dagger}(\mathbf{k}) b_{r}(\mathbf{k}) .
\end{aligned}
$$

Here we added and subtracted the term $\sum_{\mathbf{k}} \mu_{0} b_{r}^{\dagger}(\mathbf{k}) b_{r}(\mathbf{k}) \quad\left(\mu_{0} \leq 0\right)$ to avoid the difficulty of being $\mu$ positive in the condensed phases, which would lead to a singularity in the unperturbed propagator.

It should be stressed that with the canonical transformation (8) one defines bare quasiparticle states $\prod_{i} b_{r_{i}}^{\dagger}\left(\mathbf{k}_{i}\right)|0\rangle$. The time evolution of these quasiparticle states are determined by the Hamiltonian (9) which contains terms with different number of creation and destruction operators leading to the nonconservation of the total number of quasiparticles.

\section{Green's functions and correlation functions}

First we define the Green's functions as

$$
\mathcal{G}_{\gamma \delta}^{r s}(\mathbf{k}, \tau)=-\left\langle T_{\tau}\left[b_{r}^{\gamma}(\mathbf{k}, \tau) b_{s}^{\delta^{\dagger}}(\mathbf{k}, 0)\right]\right\rangle,
$$

with

$$
b_{r}^{\gamma}(\mathbf{k})= \begin{cases}b_{r}(\mathbf{k}) & , \gamma=1 \\ b_{r}^{\dagger}(-\mathbf{k}) & , \gamma=-1 .\end{cases}
$$

The Greek indices introduced here are for distinguishing between the normal and anomalous Green's functions. Automatic summation over repeated Greek indices is understood as for the Roman ones. Here and from now on $\tau$ is 
the imaginary time and $T_{\tau}$ is the $\tau$ ordering operator 40. In this symmetry breaking system because of the fact that the Hamiltonian Eq. (9) contains terms with two creation or two annihilation operators anomalous Green's functions arise (with $\gamma \delta=-1$ ). The expression (10) is the generalization of the well known normal and anomalous Green's functions in case of a complex scalar field [40,30]. The propagators (10) are periodic in $\tau$ with period $\beta \hbar$ so their Fourier series can be defined as

$$
\mathcal{G}_{\gamma \delta}^{r s}\left(\mathbf{k}, i \omega_{n}\right)=\frac{1}{\beta \hbar} \int_{0}^{\beta \hbar} d \tau e^{i \omega_{n} \tau} \mathcal{G}_{\gamma \delta}^{r s}(\mathbf{k}, \tau),
$$

where $\omega_{n}=2 n \pi / \beta \hbar$ is the Bose discrete Matsubara frequency. The spectrum of the one-particle elementary excitations can be determined as poles of the analytic continuations of the Green's functions.

The collective excitations in the system can be described with the correlation functions of the following operators:

$$
\begin{aligned}
n(\mathbf{k}) & =\sum_{\mathbf{q}} a_{r}^{\dagger}(\mathbf{k}+\mathbf{q}) a_{r}(\mathbf{q}), \\
\mathcal{F}_{z}(\mathbf{k}) & =\sum_{\mathbf{q}} a_{r}^{\dagger}(\mathbf{k}+\mathbf{q})\left(F_{z}\right)_{r s} a_{s}(\mathbf{q}), \\
\mathcal{F}_{ \pm}(\mathbf{k}) & =\sum_{\mathbf{q}} a_{r}^{\dagger}(\mathbf{k}+\mathbf{q})\left(F_{ \pm}\right)_{r s} a_{s}(\mathbf{q}), \\
\mathcal{F}_{ \pm}^{Q}(\mathbf{k}) & =\sum_{\mathbf{q}} a_{r}^{\dagger}(\mathbf{k}+\mathbf{q})\left(F_{ \pm}^{2}\right)_{r s} a_{s}(\mathbf{q}), \\
\sigma_{r s}(\mathbf{k}) & =\sum_{\mathbf{q}} a_{r}^{\dagger}(\mathbf{k}+\mathbf{q}) a_{s}(\mathbf{q}) .
\end{aligned}
$$

Here $n(\mathbf{k})$ is the particle density operator $\mathcal{F}_{z}(\mathbf{k})$ is the spin z component density operator, $\mathcal{F}_{ \pm}(\mathbf{k})$ is the density operator of the \pm 1 spin raising or lowering operator and $\mathcal{F}_{Q}^{ \pm}(\mathbf{k})$ is the density of the \pm 2 spin raising or lowering operators and at last $\sigma_{r s}(\mathbf{k})$ is the general density operator from which the other ones can be easily calculated as e.g. $n(\mathbf{k})=\sigma_{++}(\mathbf{k})+\sigma_{00}(\mathbf{k})+\sigma_{--}(\mathbf{k})$ or $\mathcal{F}_{z}(\mathbf{k})=\sigma_{++}(\mathbf{k})-\sigma_{--}(\mathbf{k})$. The different collective excitations can be found as poles of the analytical continuations of the corresponding correlation functions (defined for $\mathbf{k} \neq 0$ ), given by

$$
\begin{aligned}
& D_{n n}(\mathbf{k}, \tau)=-\left\langle T_{\tau}\left[n(\mathbf{k}, \tau) n^{\dagger}(\mathbf{k}, 0)\right]\right\rangle, \\
& D_{z z}(\mathbf{k}, \tau)=-\left\langle T_{\tau}\left[\mathcal{F}_{z}(\mathbf{k}, \tau) \mathcal{F}_{z}^{\dagger}(\mathbf{k}, 0)\right]\right\rangle, \\
& D_{n z}(\mathbf{k}, \tau)=-\left\langle T_{\tau}\left[n(\mathbf{k}, \tau) \mathcal{F}_{z}^{\dagger}(\mathbf{k}, 0)\right]\right\rangle, \\
& D_{ \pm \pm}(\mathbf{k}, \tau)=-\left\langle T_{\tau}\left[\mathcal{F}_{ \pm}(\mathbf{k}, \tau) \mathcal{F}_{ \pm}^{\dagger}(\mathbf{k}, 0)\right]\right\rangle, \\
& D_{ \pm \pm}^{Q}(\mathbf{k}, \tau)=-\left\langle T_{\tau}\left[\mathcal{F}_{ \pm}^{Q}(\mathbf{k}, \tau) \mathcal{F}_{ \pm}^{Q \dagger}(\mathbf{k}, 0)\right]\right\rangle .
\end{aligned}
$$

Note that $n^{\dagger}(\mathbf{k})=n(-\mathbf{k}), \mathcal{F}_{z}^{\dagger}(\mathbf{k})=\mathcal{F}_{z}(-\mathbf{k}), \mathcal{F}_{ \pm}^{\dagger}(\mathbf{k})=\mathcal{F}_{\mp}(-\mathbf{k})$ and $\mathcal{F}_{ \pm}^{Q \dagger}(\mathbf{k})=\mathcal{F}_{\mp}^{Q}(-\mathbf{k})$. A general correlation function can also be defined as

$$
D_{r^{\prime} s^{\prime}}^{s r}(\mathbf{k}, \tau)=-\left\langle T_{\tau}\left[\sigma_{r s}(\mathbf{k}, \tau) \sigma_{s^{\prime} r^{\prime}}(-\mathbf{k}, 0)\right]\right\rangle,
$$

from what the above ones can be calculated as:

$$
\begin{aligned}
& D_{n n}(\mathbf{k}, \tau)=\sum_{r, s} D_{s s}^{r r}(\mathbf{k}, \tau), \\
& D_{z z}(\mathbf{k}, \tau)=\sum_{r, s} r s D_{s s}^{r r}(\mathbf{k}, \tau), \\
& D_{n z}(\mathbf{k}, \tau)=\sum_{r, s} s D_{s s}^{r r}(\mathbf{k}, \tau), \\
& D_{++}(\mathbf{k}, \tau)=2\left[D_{+0}^{0+}(\mathbf{k}, \tau)+D_{0-}^{0+}(\mathbf{k}, \tau)+D_{+0}^{-0}(\mathbf{k}, \tau)+D_{0-}^{-0}(\mathbf{k}, \tau)\right], \\
& D_{--}(\mathbf{k}, \tau)=2\left[D_{-0}^{0-}(\mathbf{k}, \tau)+D_{0+}^{0-}(\mathbf{k}, \tau)+D_{-0}^{+0}(\mathbf{k}, \tau)+D_{0+}^{+0}(\mathbf{k}, \tau)\right], \\
& D_{++}^{Q}(\mathbf{k}, \tau)=4 D_{+-}^{-+}(\mathbf{k}, \tau), \\
& D_{--}^{Q}(\mathbf{k}, \tau)=4 D_{-+}^{+-}(\mathbf{k}, \tau) .
\end{aligned}
$$


All of these correlation functions are also periodic in $\tau$ with period $\beta \hbar$, so their Fourier transforms can be defined analogously to Eq. (12) and the appearing Matsubara frequencies are also the same as for the Green's functions.

We will also apply anomalous correlation functions

$$
\begin{aligned}
& A_{a \alpha}^{s r}(\mathbf{k}, \tau)=-\left\langle T_{\tau}\left[\sigma_{r s}(\mathbf{k}, \tau) b_{a}^{\alpha^{\dagger}}(\mathbf{k}, 0)\right]\right\rangle, \\
& A_{r^{\prime} s^{\prime}}^{a \alpha}(\mathbf{k}, \tau)=-\left\langle T_{\tau}\left[b_{a}^{\alpha}(\mathbf{k}, \tau) \sigma_{s^{\prime} r^{\prime}}(-\mathbf{k}, 0)\right]\right\rangle,
\end{aligned}
$$

which have zero value in the symmetric phase (i.e. for a noncondensed system).

Though the one-particle Green's functions are the autocorrelation functions of the order parameter field operator, we preserve in the following the term correlation function for the other correlation functions introduced above.

\section{GENERAL FORMALISM}

In this section the general formalism is worked out. First symmetry properties of the functions defined in the previous section will be discussed. Next perturbation theory will be presented and then we will turn our attention to relations valid in all orders of perturbation theory.

\section{A. Symmetry properties}

Due to the rotational symmetry in the coordinate space the Green's functions (10) and all the correlation functions (14) and (16) depend only on the modulus of the momentum.

For the Green's functions the following symmetry properties can be derived. Since (in absence of a magnetic filed) the Green's functions (10) are real and the cyclic property of trace

$$
\begin{array}{r}
\mathcal{G}_{\gamma \delta}^{r s}(\mathbf{k}, \tau)=-\left\langle T_{\tau}\left[b_{r}^{\gamma}(\mathbf{k}, \tau) b_{s}^{\delta^{\dagger}}(\mathbf{k}, 0)\right]\right\rangle=-\left\langle T_{\tau}\left[b_{r}^{\gamma}(\mathbf{k}, \tau) b_{s}^{\delta^{\dagger}}(\mathbf{k}, 0)\right]^{\dagger}\right\rangle \\
=-\left\langle T_{\tau}\left[b_{s}^{\delta}(\mathbf{k}, \tau) b_{r}^{\gamma^{\dagger}}(\mathbf{k}, 0)\right]\right\rangle=\mathcal{G}_{\delta \gamma}^{s r}(\mathbf{k}, \tau)
\end{array}
$$

holds. Furthermore the time displacement symmetry of Hamiltonian systems, the $b_{r}^{\gamma}(\mathbf{k})=b_{r}^{-\gamma \dagger}(-\mathbf{k})$ relation and that after $T_{\tau}$ the order of the bosonic operators are irrelevant lead to

$$
\begin{array}{r}
\mathcal{G}_{\gamma \delta}^{r s}(\mathbf{k}, \tau)=-\left\langle T_{\tau}\left[b_{r}^{\gamma}(\mathbf{k}, \tau) b_{s}^{\delta^{\dagger}}(\mathbf{k}, 0)\right]\right\rangle=-\left\langle T_{\tau}\left[b_{s}^{\delta^{\dagger}}(\mathbf{k}, 0) b_{r}^{\gamma}(\mathbf{k}, \tau)\right]\right\rangle \\
=-\left\langle T_{\tau}\left[b_{s}^{\delta^{\dagger}}(\mathbf{k},-\tau) b_{r}^{\gamma}(\mathbf{k}, 0)\right]\right\rangle=-\left\langle T_{\tau}\left[b_{s}^{-\delta}(-\mathbf{k},-\tau) b_{r}^{-\gamma^{\dagger}}(-\mathbf{k}, 0)\right]\right\rangle \\
=\mathcal{G}_{-\delta,-\gamma}^{s r}(-\mathbf{k},-\tau) .
\end{array}
$$

On the basis of the above equalities one finds

$$
\mathcal{G}_{\gamma \delta}^{r s}(\mathbf{k}, \tau)=\mathcal{G}_{\gamma \delta}^{r s}(k, \tau)=\mathcal{G}_{\delta \gamma}^{s r}(k, \tau)=\mathcal{G}_{-\delta,-\gamma}^{s r}(k,-\tau)=\mathcal{G}_{-\gamma,-\delta}^{r s}(k,-\tau) .
$$

For the generalized density correlation functions of Eq. (141) the following symmetry relations can be derived:

$$
\begin{array}{r}
-D_{r^{\prime} s^{\prime}}^{s r}(\mathbf{k}, \tau)=\left\langle T_{\tau}\left[\sigma_{r s}(\mathbf{k}, \tau) \sigma_{s^{\prime} r^{\prime}}(-\mathbf{k}, 0)\right]^{\dagger}\right\rangle=\left\langle T_{\tau}\left[\sigma_{s^{\prime} r^{\prime}}^{\dagger}(-\mathbf{k}, 0) \sigma_{r s}^{\dagger}(\mathbf{k},-\tau)\right]\right\rangle \\
=\left\langle T_{\tau}\left[\sigma_{r^{\prime} s^{\prime}}(\mathbf{k}, \tau) \sigma_{s r}(-\mathbf{k}, 0)\right]\right\rangle=-D_{r s}^{s^{\prime} r^{\prime}}(\mathbf{k}, \tau)
\end{array}
$$

and

$$
\begin{array}{r}
-D_{r^{\prime} s^{\prime}}^{s r}(\mathbf{k}, \tau)=\left\langle T_{\tau}\left[\sigma_{r s}(\mathbf{k}, \tau) \sigma_{s^{\prime} r^{\prime}}(-\mathbf{k}, 0)\right]\right\rangle=\left\langle T_{\tau}\left[\sigma_{s^{\prime} r^{\prime}}(-\mathbf{k}, 0) \sigma_{r s}(\mathbf{k}, \tau)\right]\right\rangle \\
=\left\langle T_{\tau}\left[\sigma_{s^{\prime} r^{\prime}}(-\mathbf{k},-\tau) \sigma_{r s}(\mathbf{k}, 0)\right]\right\rangle=-D_{s r}^{r^{\prime} s^{\prime}}(-\mathbf{k},-\tau),
\end{array}
$$

where we further used that $\sigma_{r s}^{\dagger}(\mathbf{k})=\sigma_{s r}(-\mathbf{k})$. Combining Eqs. (20) and (21) together one arrives at

$$
D_{r^{\prime} s^{\prime}}^{s r}(\mathbf{k}, \tau)=D_{r^{\prime} s^{\prime}}^{s r}(k, \tau)=D_{r s}^{s^{\prime} r^{\prime}}(k, \tau)=D_{s r}^{r^{\prime} s^{\prime}}(k,-\tau)=D_{s^{\prime} r^{\prime}}^{r s}(k,-\tau)
$$


For the anomalous correlation functions the two symmetry properties can be derived in the same way. The first one cames form the fact that the expectation values here are also real

$$
A_{a \alpha}^{s r}(\mathbf{k}, \tau)=-\left\langle T_{\tau}\left[\sigma_{r s}(\mathbf{k}, \tau) b_{a}^{\alpha^{\dagger}}(\mathbf{k}, 0)\right]^{\dagger}\right\rangle=-\left\langle T_{\tau}\left[b_{a}^{\alpha}(\mathbf{k}, \tau) \sigma_{s r}(-\mathbf{k}, 0)\right]\right\rangle=A_{r s}^{a \alpha}(\mathbf{k}, \tau) .
$$

The second one can derived using the invariance of the trace under cyclic permutations and that the order is irrelevant behind an ordering operator:

$$
A_{a \alpha}^{s r}(-\mathbf{k},-\tau)=-\left\langle T_{\tau}\left[\sigma_{r s}(-\mathbf{k},-\tau) b_{a}^{\alpha^{\dagger}}(-\mathbf{k}, 0)\right]\right\rangle=-\left\langle T_{\tau}\left[b_{a}^{\alpha^{\dagger}}(-\mathbf{k}, \tau) \sigma_{r s}(-\mathbf{k}, 0)\right]\right\rangle=A_{s r}^{a,-\alpha}(\mathbf{k}, \tau)
$$

Combining Eq. (23) and Eq. (24) together results in (also using that the momentum dependence comes from $k=|\mathbf{k}|$ )

$$
A_{a \alpha}^{s r}(\mathbf{k}, \tau)=A_{a \alpha}^{s r}(k, \tau)=A_{r s}^{a \alpha}(k, \tau)=A_{s r}^{a,-\alpha}(k,-\tau)=A_{a,-\alpha}^{r s}(k,-\tau) .
$$

\section{B. Perturbation theory}

To calculate the averages of the grand canonical ensemble with the Hamiltonian (9) one can use the methods of the finite temperature many body physics 40 .

The Hamiltonian of the non interacting system is the first term in Eq. (9):

$$
\mathcal{H}_{0}=\sum_{\mathbf{k}}\left(e_{\mathbf{k}}-\mu_{0}\right) b_{r}^{\dagger}(\mathbf{k}) b_{r}(\mathbf{k})
$$

which defines free Green's functions:

$$
\mathcal{G}_{(0) \gamma \delta}^{r s}\left(\mathbf{k}, i \omega_{n}\right)=\frac{\delta_{r s} \delta_{\gamma \delta}}{\gamma i \omega_{n}-\hbar^{-1}\left(e_{\mathbf{k}}-\mu_{0}\right)}
$$

This will be symbolized as a line or a line with an arrow if the Greek indices are specified as seen in Fig. 11. The full Green's functions will be symbolized with double lines as shown in Fig. 2. The terms in the Hamiltonian Eq. (9) without any filed operator can be disregarded for our purposes. Among the interaction terms there is one containing four field operators and corresponding to a scattering of two quasi-particles which are noncondensed before and after the collision as well. There are four interaction terms containing three field operators and six terms containing two field operators, corresponding to scattering processes involving both condensate and noncondensate atoms. There are interaction terms containing one field operator describing scattering processes involving three condensate and one noncondensate atoms. These interaction terms will be graphically represented as shown in Fig. 3. There is one remaining term with one field operator and the last term with two operators corresponding to vertices with one and two legs as seen in Fig. 4 .

$$
-\mathcal{G}_{(0) \gamma \delta}^{r s}\left(\mathbf{k}, i \omega_{n}\right): \frac{r}{\gamma} \delta \quad, \quad-\mathcal{G}_{(0) 1,1}^{r s}\left(\mathbf{k}, i \omega_{n}\right): \stackrel{r}{s}, \quad-\mathcal{G}_{(0)-1,-1}^{r s}\left(\mathbf{k}, i \omega_{n}\right): \stackrel{r}{\longrightarrow}
$$

FIG. 1. The graphical representation of the free propagators

$$
\begin{aligned}
& -\mathcal{G}_{\gamma \delta}^{r s}\left(\mathbf{k}, i \omega_{n}\right): \frac{r}{\gamma} \frac{s}{\delta}, \quad-\mathcal{G}_{1,1}^{r s}\left(\mathbf{k}, i \omega_{n}\right): \stackrel{r}{=} \stackrel{s}{=}, \quad-\mathcal{G}_{1,-1}^{r s}\left(\mathbf{k}, i \omega_{n}\right): \underbrace{r}, \\
& -\mathcal{G}_{-1,1}^{r s}\left(\mathbf{k}, i \omega_{n}\right): \stackrel{r}{=} \stackrel{s}{=}, \quad-\mathcal{G}_{-1,-1}^{r s}\left(\mathbf{k}, i \omega_{n}\right): \stackrel{r}{=} \stackrel{s}{=} .
\end{aligned}
$$

FIG. 2. The graphical representation of the full propagators 


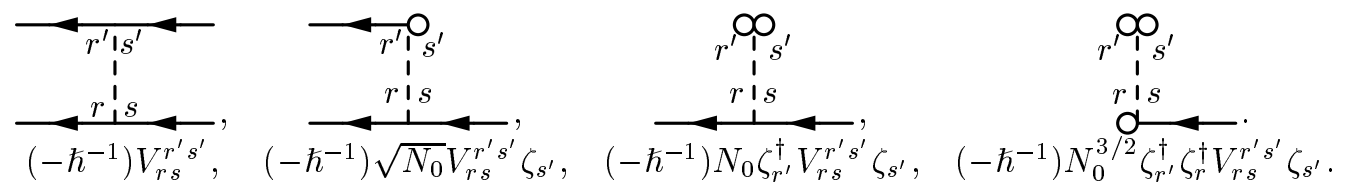

FIG. 3. The Feynman graphs of a few interaction processes involving zero, one, two and three condensate atoms

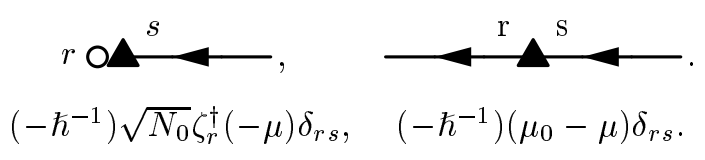

FIG. 4. The Feynamn graphs of the non interaction processes

The Green's functions can be expanded to perturbation series in the usual way. Rearranging these series one arrives at the generalized Dyson-Beliaev equations 40, 30,41:

$$
\mathcal{G}_{\gamma \delta}^{r s}\left(\mathbf{k}, i \omega_{n}\right)=\mathcal{G}_{(0) \gamma \delta}^{r s}\left(\mathbf{k}, i \omega_{n}\right)+\mathcal{G}_{(0) \gamma \rho}^{r r^{\prime}}\left(\mathbf{k}, i \omega_{n}\right) \Sigma_{\rho \sigma}^{r^{\prime} s^{\prime}}\left(\mathbf{k}, i \omega_{n}\right) \mathcal{G}_{\sigma \delta}^{s^{\prime} s}\left(\mathbf{k}, i \omega_{n}\right),
$$

where $\Sigma_{\gamma \delta}^{r s}$ is the self-energy, the contribution of those graphs which are one-particle irreducible (cannot be split to two by cutting a single one-particle line) and connect to two external lines with indices $(r, \gamma)$ and $(s, \delta)$. This equation is graphically represented in Fig. 5. The symmetry properties of the Green's functions of Eq. (19) stand for the self-energies as well.

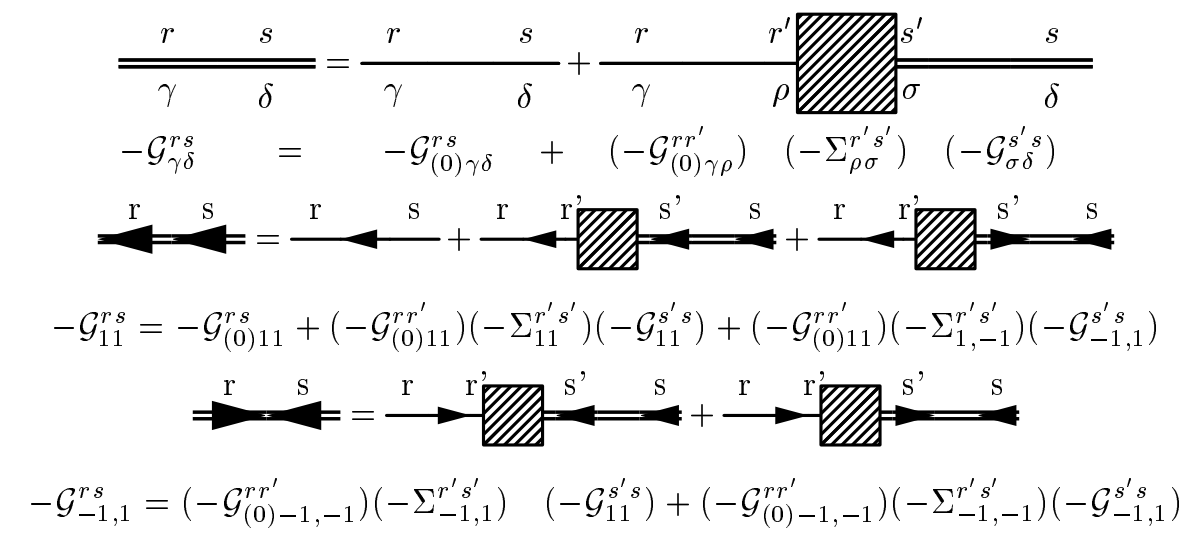

FIG. 5. The graphical symbolization of the Dyson-Beliaev equations, the hatched squares represent the one-particle irreducible graphs

Similar rearrangements can be carried out for the perturbation series of the generalized density correlation functions (14t). One can introduce their proper parts (the polarization parts), the contribution of those graphs (the polarization graphs) which cannot be split by two with cutting a single interaction line, that is they are interaction line irreducible. Then the equations determining these correlation functions read as:

$$
D_{r^{\prime} s^{\prime}}^{s r}\left(\mathbf{k}, i \omega_{n}\right)=\hbar \Pi_{r^{\prime} s^{\prime}}^{s r}\left(\mathbf{k}, i \omega_{n}\right)+\Pi_{a b}^{s r}\left(\mathbf{k}, i \omega_{n}\right) V_{c d}^{b a} D_{r^{\prime} s^{\prime}}^{d c}\left(\mathbf{k}, i \omega_{n}\right) .
$$

The Feynman graph corresponding to this equation is shown in Fig. 6, where the generalized density correlation functions are represented as boxes which can connect to two interaction lines and their proper parts are represented as grey polygons also able to connect to two interaction lines. The proper parts satisfy the same symmetry properties as the generalized correlation functions (see Eq. (22)). 


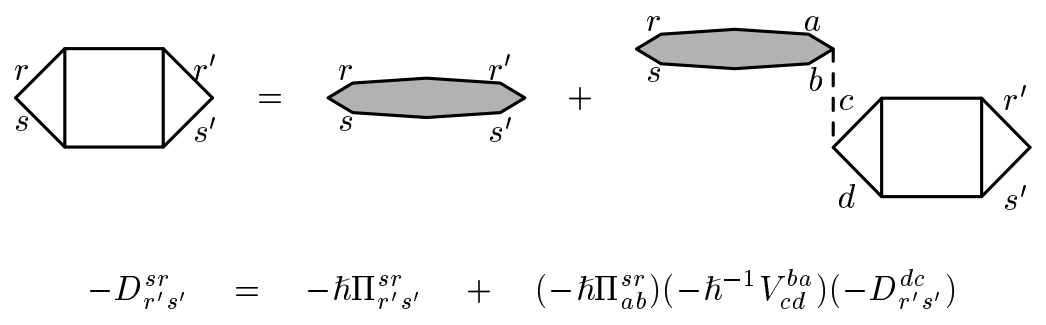

FIG. 6. The graphical representation of Eq. (29). The gray polygon represents the proper graphs

Above the critical temperature (or for a noncondensed system), where $\left\langle a_{r}(\mathbf{k})\right\rangle=0$ for all $\mathbf{k}$, the anomalous correlation functions (16) are zero. In this case the self-energies, which are irreducible by definition are proper as well and the polarization parts, which are proper by definition are irreducible as well. In the Bose condensed phase the appearance of the anomalous averages $\left\langle a_{r}(\mathbf{0})\right\rangle \neq 0$ leads to the nonzero value of the anomalous correlation functions and to a situation where the self-energies are no longer proper and the polarization parts are no longer irreducible. However, they can be separated such as

$$
\begin{aligned}
\Sigma_{\gamma \delta}^{r s} & =\widetilde{\Sigma}_{\gamma \delta}^{r s}+M_{\gamma \delta}^{r s}, \\
\Pi_{r^{\prime} s^{\prime}}^{s r} & =\Pi_{r^{\prime} s^{\prime}}^{(r) s r}+\Pi_{r^{\prime} s^{\prime}}^{(s) s r}
\end{aligned}
$$

where $\widetilde{\Sigma}$ is the contribution of those self-energy graphs which are proper, while the graphs contributing to $M$ will be improper, and similarly $\Pi^{(r)}$ is the contribution of those polarization graphs which are irreducible as well and $\Pi^{(s)}$ is the contribution of the reducible polarization graphs. The separations (30a) and (30b) are the starting steps toward the dielectric formalism. See for the scalar gas Ref. [31.30].

It can be directly seen from the perturbation series of the anomalous correlation functions (16a) can be decomposed such a way that

$$
A_{a \alpha}^{s r}\left(\mathbf{k}, i \omega_{n}\right)=\Lambda_{c \gamma}^{s r}\left(\mathbf{k}, i \omega_{n}\right) \mathcal{G}_{\gamma \alpha}^{c a}\left(\mathbf{k}, i \omega_{n}\right)
$$

where $\mathcal{G}_{\gamma \alpha}^{c a}$ is the one-particle Green's function and $\Lambda_{c \gamma}^{s r}$ is the anomalous vertex, which is the sum of the irreducible contributions of those graphs with one incoming interaction and one incoming particle line. These anomalous vertex functions can be expressed with the use their proper parts and the irreducible and proper parts of the density correlation functions as seen in Fig. 7.

$$
\Lambda_{a \alpha}^{s r}\left(\mathbf{k}, i \omega_{n}\right)=\widetilde{\Lambda}_{a \alpha}^{s r}\left(\mathbf{k}, i \omega_{n}\right)+\Pi_{c d}^{(r) s r}\left(\mathbf{k}, i \omega_{n}\right) V_{e f}^{d c} \Lambda_{a \alpha}^{f e}\left(\mathbf{k}, i \omega_{n}\right) .
$$

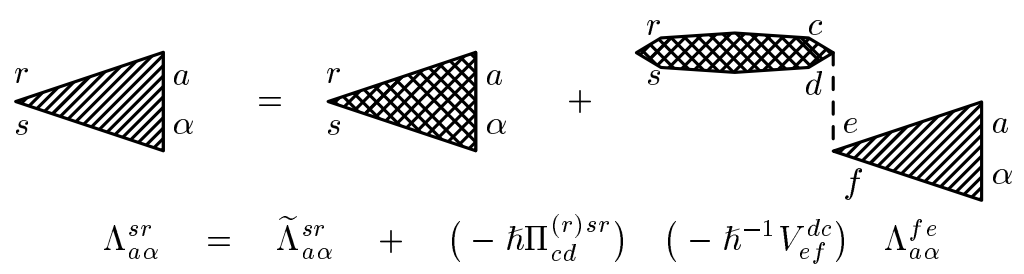

FIG. 7. The graphical symbolization of building up of the anomalous vertex from proper parts

\section{The determination of the chemical potential and the treatment of the non-interaction self-energy vertex}

As pointed out earlier, with the introduction of the new set of creation and destruction operators with Eqs. (8a) and (8b) one can derive the relation between the condensate density and the chemical potential from the requirement that $\left\langle b_{r}(\mathbf{k})\right\rangle=\left\langle b_{r}^{\dagger}(\mathbf{k})\right\rangle=0$. Using perturbation theory for the evaluation of these expressions one can arrive at equations 
which can be symbolized as seen in Fig. 8. One can notice that a similar rearrangement is possible as was made with the Green's functions which leads to:

$$
\left\langle b_{r}^{\gamma}(0,0)\right\rangle=\Sigma_{0 \delta}^{s}(0,0) \mathcal{G}_{\delta \gamma}^{s r}(0,0),
$$

where $\Sigma_{0 \delta}^{s}$ is the sum of those irreducible graphs which has only one incoming (outgoing) lines. Using the symmetry properties of the Green's functions one can easily derive that the consistency condition is equivalent to

$$
\begin{gathered}
\Sigma_{0 \gamma}^{s}(0,0)=0 . \\
\left\langle b_{r}^{\dagger}(0)\right\rangle=s^{\prime} s^{r}+a^{\prime} \propto s^{\prime} \\
\left\langle b_{r}^{\dagger}(0)\right\rangle=\frac{-1}{\hbar}(-\mu) \sqrt{N_{0}} \zeta_{s}^{\dagger}\left(-\mathcal{G}_{(0) 1,1}^{s r}\right)+\frac{-1}{\hbar} N_{0}^{3 / 2} \zeta_{a}^{\dagger} \zeta_{a^{\prime}}^{\dagger} V_{a s}^{a^{\prime} s^{\prime}} \zeta_{s^{\prime}}\left(-\mathcal{G}_{(0) 1,1}^{s r}\right)+\ldots
\end{gathered}
$$

FIG. 8. The beginning of the perturbation series of the consistency condition

The non interaction self-energy vertex corresponding to the last term in the Hamiltonian of Eq. (9) is to be calculated in each order of perturbation theory. Since this vertex is proper and diagonal in spin and anomalous indices it is part of the proper self-energy as illustrated in Fig. 9.

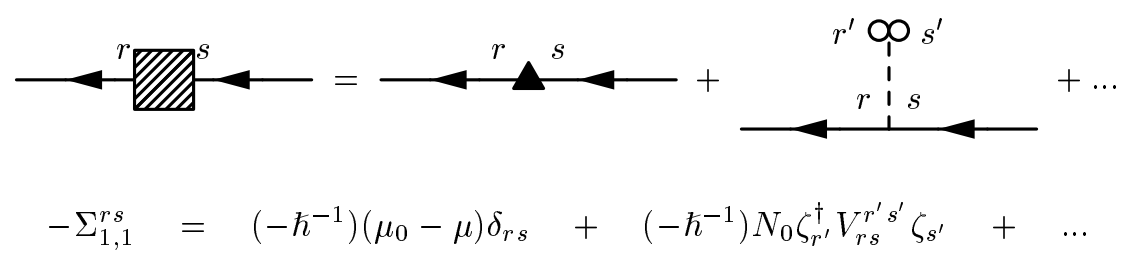

FIG. 9. The self-energy with the non interaction vertex

\section{Rotational symmetry and spin transfer decomposition}

Since rotational symmetry along the $\mathrm{z}$ axis is not broken the $\mathrm{z}$ component of the total angular momentum is conserved which results for $\mathcal{G}_{\gamma \delta}^{r s}$ and $\Sigma_{\gamma \delta}^{r s}$ in $\gamma r-\delta s=(\gamma-\delta) \zeta_{r}^{\dagger}\left(F_{z}\right)_{r s} \zeta_{s}$ or symbolically:
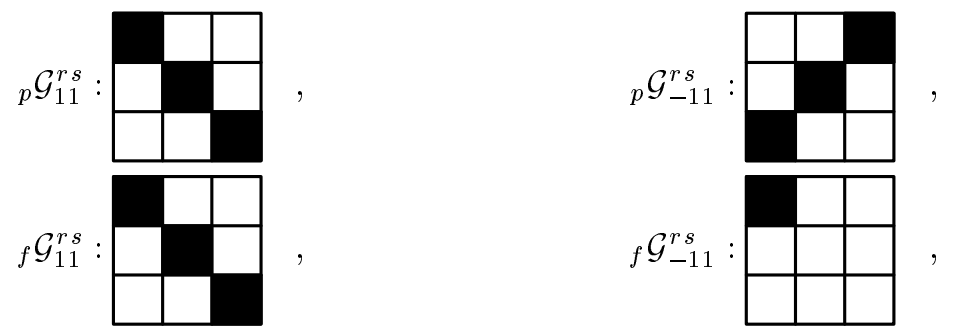

FIG. 10. The spin conservation property of the Green's functions. Here ${ }_{p} \mathcal{G}$ denotes the Green's functions of the polar case while ${ }_{f} \mathcal{G}$ denotes the Green's functions of the ferromagnetic case. A black box refers to a non zero element and we used $\zeta=(0,1,0)^{T}$ for the polar case and $\zeta=(1,0,0)^{T}$ for the ferromagnetic case specifying the condensate 
As a consequence of these symmetry relations Eq. (28) can be separated to three equations according to the number $n=[\gamma(c-r)+\delta(c-s)] / 2$, with $c=\zeta_{r}^{\dagger}\left(F_{z}\right)_{r s} \zeta_{s}$ specifying the spin carried by the propagator relative to the spin of the atom in the condensate. For this reason let us define the following matrices for the polar case

$$
\begin{aligned}
{ }^{0} \mathcal{G}_{\gamma \delta} & =\left[\begin{array}{cc}
\mathcal{G}_{1,1}^{00} & \mathcal{G}_{1,-1}^{00} \\
\mathcal{G}_{-1,1}^{00} & \mathcal{G}_{-1,-1}^{00}
\end{array}\right]_{\gamma \delta}, & { }^{0} \Sigma_{\gamma \delta} & =\left[\begin{array}{cc}
\Sigma_{1,1}^{00} & \Sigma_{1,-1}^{00} \\
\Sigma_{-1,1}^{00} & \Sigma_{-1,-1}^{00}
\end{array}\right]_{\gamma \delta}, \\
{ }^{+} \mathcal{G}_{\gamma \delta} & =\left[\begin{array}{cc}
\mathcal{G}_{1,1}^{--} & \mathcal{G}_{1,-1}^{-+} \\
\mathcal{G}_{-1,1}^{+-} & \mathcal{G}_{-1,-1}^{++}
\end{array}\right]_{\gamma \delta}, & { }^{+} \Sigma_{\gamma \delta} & =\left[\begin{array}{cc}
\Sigma_{1,1}^{--} & \Sigma_{1,-1}^{-+} \\
\Sigma_{-1,1}^{+-} & \Sigma_{-1,-1}^{++}
\end{array}\right]_{\gamma \delta} \\
{ }^{-} \mathcal{G}_{\gamma \delta} & =\left[\begin{array}{cc}
\mathcal{G}_{1,1}^{++} & \mathcal{G}_{1,-1}^{+-} \\
\mathcal{G}_{-1,1}^{-+} & \mathcal{G}_{-1,-1}^{-1}
\end{array}\right]_{\gamma \delta}, & { }^{-} \Sigma_{\gamma \delta} & =\left[\begin{array}{cc}
\Sigma_{1,1}^{++} & \Sigma_{1,-1}^{+-} \\
\Sigma_{-1,1}^{-+} & \Sigma_{-1,-1}^{-,}
\end{array}\right]_{\gamma \delta}
\end{aligned}
$$

where the indices in the upper left corner correspond to the number $n$. For the ferromagnetic one let us define the following quantities

$$
\begin{aligned}
{ }^{0} \mathcal{G}_{\gamma \delta} & =\left[\begin{array}{ll}
\mathcal{G}_{1,1}^{++} & \mathcal{G}_{1,-1}^{++} \\
\mathcal{G}_{-1,1}^{++} & \mathcal{G}_{-1,-1}^{++}
\end{array}\right]_{\gamma \delta}, & { }^{0} \Sigma_{\gamma \delta} & =\left[\begin{array}{cc}
\Sigma_{1,1}^{++} & \Sigma_{1,-1}^{++} \\
\Sigma_{-1,1}^{++} & \Sigma_{-1,-1}^{++}
\end{array}\right]_{\gamma \delta}, \\
{ }^{+} \mathcal{G} & =\mathcal{G}_{1,1}^{00}, & { }^{+} & =\Sigma_{1,1}^{00}, \\
{ }^{-} \mathcal{G} & =\mathcal{G}_{-1,-1}^{00}, & - & =\Sigma_{-1,-1}^{00}, \\
{ }^{Q} \mathcal{G} & =\mathcal{G}_{1,1}^{--}, & Q_{\Sigma} & =\Sigma_{1,1}^{--}, \\
{ }^{-Q} \mathcal{G} & =\mathcal{G}_{-1,-1}^{--}, & -Q_{\Sigma} & =\Sigma_{-1,-1}^{--} .
\end{aligned}
$$

The indices in the upper left corner correspond to the number $n$ except for the quadrupolar spin transfer $n=2$ and similarly $-Q$ is for $n=-2$. (Note, that there is no quadrupolar Green's function for the polar case because the value of $n$ can not be \pm 2 here.) The later modes of the ferromagnetic case $(+,-, Q,-Q)$ differ from all the other modes since for these cases spin conservation forbids the existence of anomalous Green's functions and self energies. With these definitions one can explicitly construct the Green's functions. The propagators corresponding to different spin transfers will be called different modes in the following. For all three modes of the polar case $(n=0,+,-)$ and for the first mode of the ferromagnetic case $(n=0)$ the Green's functions are

$$
{ }^{n} \mathcal{G}_{\alpha \gamma}=\frac{\delta_{\alpha, \gamma}\left(\alpha i \omega_{n}+\hbar^{-1} \epsilon_{\mathbf{k}}\right)+\alpha \gamma \Sigma_{-\gamma,-\alpha}}{\left(i \omega_{n}-\hbar^{-1} \epsilon_{\mathbf{k}}-\Sigma_{1,1}\right)\left(i \omega_{n}+\hbar^{-1} \epsilon_{\mathbf{k}}+\Sigma_{-1,-1}\right)+\Sigma_{-1,1} \Sigma_{1,-1}} \equiv \frac{{ }^{n} N_{\alpha, \gamma}}{{ }^{n} \Delta}
$$

where $\epsilon_{\mathbf{k}}=e_{\mathbf{k}}-\mu_{0}$ is introduced. This equation defines the quantities ${ }^{n} N_{\alpha \gamma}$ and ${ }^{n} \Delta$ for the first four modes. For the last four modes of the ferromagnetic case one can get

$$
\begin{aligned}
& { }^{ \pm} \mathcal{G}=\frac{1}{ \pm i \omega_{n}-\hbar^{-1} \epsilon_{\mathbf{k}}-{ }^{ \pm \Sigma}}, \\
& { }^{ \pm Q} \mathcal{G}=\frac{1}{ \pm i \omega_{n}-\hbar^{-1} \epsilon_{\mathbf{k}}-{ }^{ \pm Q \Sigma}} .
\end{aligned}
$$

Sometimes it will be practical to cast the formulas into a most concise form. This can be achieved by introducing the following formal definitions:

$$
\begin{aligned}
{ }^{+} \mathcal{G}_{\gamma \delta} & =\left[\begin{array}{cc}
\mathcal{G}_{1,1}^{00} & 0 \\
0 & 0
\end{array}\right]_{\gamma \delta}, & { }^{+} \Sigma_{\gamma \delta} & =\left[\begin{array}{cc}
\Sigma_{1,1}^{00} & 0 \\
0 & 0
\end{array}\right]_{\gamma \delta}, \\
{ }^{-} \mathcal{G}_{\gamma \delta} & =\left[\begin{array}{cc}
0 & 0 \\
0 & \mathcal{G}_{-1,-1}^{00}
\end{array}\right]_{\gamma \delta}, & { }^{-} \Sigma_{\gamma \delta} & =\left[\begin{array}{cc}
0 & 0 \\
0 & \Sigma_{-1,-1}^{00}
\end{array}\right]_{\gamma \delta}
\end{aligned}
$$

with the help of which equation (38a) takes the form of Eq. (37), i.e., the validity of Eq. (37) is extended for $n=0,+,-$ for the ferromagnetic phase as well.

The conservation of the $\mathrm{z}$ component of the spin for these $D_{r^{\prime} s^{\prime}}^{s r}$ correlation functions means that $r-s=r^{\prime}-s^{\prime}$ which holds for the proper parts as well. And as a consequence Eq. (29) also decouples into three ordinary matrix equations according to the specific spin transfer. For this reason it is useful to define the following matrices: 
- For 0 spin transfer:

$$
\begin{aligned}
& \left({ }^{0} \underline{\underline{D}}\right)_{a b}:=D_{b b}^{a a}=\left[\begin{array}{ccc}
D_{++}^{++} & D_{00}^{++} & D_{--}^{++} \\
D_{++}^{00} & D_{00}^{00} & D_{--}^{00} \\
D_{+-}^{-+} & D_{00}^{--} & D_{--}^{--}
\end{array}\right]_{a b}, \\
& \left({ }^{0} \underline{\underline{ }}\right)_{a b}:=\Pi_{b b}^{a a}=\left[\begin{array}{ccc}
\Pi_{++}^{++} & \Pi_{00}^{++} & \Pi_{-+}^{++} \\
\Pi_{++}^{00} & \Pi_{00}^{00} & \Pi_{--}^{00} \\
\Pi_{++}^{--} & \Pi_{00}^{--} & \Pi_{--}^{--}
\end{array}\right]_{a b}, \\
& \left({ }^{0} \underline{\underline{C}}\right)_{a b}:=V_{b b}^{a a}=\left[\begin{array}{ccc}
c_{n}+c_{s} & c_{n} & c_{n}-c_{s} \\
c_{n} & c_{n} & c_{n} \\
c_{n}-c_{s} & c_{n} & c_{n}+c_{s}
\end{array}\right]_{a b},
\end{aligned}
$$

where no automatic summation is understood now. From Eq. (29) one obtains

$$
{ }^{0} \underline{\underline{D}}=\hbar^{0} \underline{\underline{\Pi}}+{ }^{0} \underline{\underline{\Pi}}{ }^{0} \underline{\underline{C}}{ }^{0} \underline{\underline{D}}
$$

- For +1 spin transfer:

$$
\begin{aligned}
& \left({ }^{+} \underline{\underline{D}}\right)_{a b}:=D_{b+1, b}^{a, a+1}=\left[\begin{array}{ll}
D_{+0}^{0+} & D_{0-}^{0+} \\
D_{+0}^{-0} & D_{0-}^{-0}
\end{array}\right]_{a b}, \\
& \left({ }^{+} \underline{\underline{ }}\right)_{a b}:=\Pi_{b+1, b}^{a, a+1}=\left[\begin{array}{ll}
\Pi_{+0}^{0+} & \Pi_{0-}^{0+} \\
\Pi_{+0}^{-0} & \Pi_{0-}^{-0}
\end{array}\right]_{a b} \\
& \left({ }^{+} \underline{\underline{C}}\right)_{a b}:=V_{b+1, b}^{a, a+1}=\left[\begin{array}{ll}
c_{s} & c_{s} \\
c_{s} & c_{s}
\end{array}\right]_{a b}
\end{aligned}
$$

and the resulting matrix equation is:

$$
{ }^{+} \underline{\underline{D}}=\hbar^{+} \underline{\underline{\Pi}}+{ }^{+} \underline{\underline{\Pi}}^{+} \underline{\underline{C}}^{+} \underline{\underline{D}}
$$

- For -1 spin transfer:

$$
\begin{aligned}
& \left({ }^{-} \underline{\underline{D}}\right)_{a b}:=D_{b, b+1}^{a+1, a}=\left[\begin{array}{ll}
D_{0+}^{+0} & D_{-0}^{+0} \\
D_{0+}^{0-} & D_{-0}^{0-}
\end{array}\right]_{a b}, \\
& \left(^{-} \underline{\underline{\underline{\Pi}}}\right)_{a b}:=\Pi_{b, b+1}^{a+1, a}=\left[\begin{array}{ll}
\Pi_{0+}^{+0} & \Pi_{-0}^{+0} \\
\Pi_{0+}^{0-} & \Pi_{-0}^{0-}
\end{array}\right]_{a b} \\
& \left(^{-} \underline{\underline{C}}\right)_{a b}:=V_{b, b+1}^{a+1, a}=\left[\begin{array}{ll}
c_{s} & c_{s} \\
c_{s} & c_{s}
\end{array}\right]_{a b}
\end{aligned}
$$

and similarly

$$
{ }^{-} \underline{\underline{D}}=\hbar^{-} \underline{\underline{\Pi}}+{ }^{-} \underline{\underline{\Pi}}^{-} \underline{\underline{C}}^{-} \underline{\underline{D}}
$$

- For the \pm 2 spin transfer case from the fact that $V_{-+}^{+-}=V_{+-}^{-+}=0$ the following results can be obtained

$$
\begin{aligned}
& D_{+-}^{-+}=\hbar \Pi_{+-}^{-+} \\
& D_{-+}^{+-}=\hbar \Pi_{-+}^{+-} .
\end{aligned}
$$

As a consequence of Eq. 22) ${ }^{0} \underline{\underline{D}},{ }^{ \pm} \underline{\underline{D}}$ (and their proper parts) are symmetric matrices, furthermore ${ }^{0} \underline{\underline{D}}\left(k, i \omega_{n}\right)=$ ${ }^{0} \underline{\underline{D}}\left(k,-i \omega_{n}\right)$ and ${ }^{+} \underline{\underline{D}}\left(k, i \omega_{n}\right)=-\underline{\underline{\underline{D}}}\left(k,-i \omega_{n}\right)$. This means that the generalized density correlation functions for zero spin transfer are completely symmetric under time reversal.

Equation (32) also decouples according to the amount of spin transferred.

$$
\begin{aligned}
& { }^{0} \underline{\Lambda}_{\alpha}={ }^{0} \underline{\widetilde{\Lambda}}_{\alpha}+{ }^{0} \underline{\underline{\Pi}}^{(r)}{ }^{0} \underline{\underline{C}}{ }^{0} \underline{\Lambda}_{\alpha}, \\
& { }^{ \pm} \underline{\Lambda}_{\alpha}={ }^{ \pm} \underline{\widetilde{\Lambda}}_{\alpha}+{ }^{ \pm} \underline{\underline{\Pi}}^{(r)}{ }^{ \pm} \underline{\underline{C}}{ }^{ \pm} \underline{\Lambda}_{\alpha},
\end{aligned}
$$


where the introduced $\underline{\Lambda}_{\alpha}$ vectors are different for the polar and for the ferromagnetic cases since the allowed spin projection of the incoming (outgoing) one-particle is determined by the rule of spin conservation and the spin projection of the condensate. It results that for a given spin transfer the spin projection of the incoming (outgoing) particle can take only one value (others are forbidden by spin conservation). So for the polar case (where $\left.c=\zeta_{r}^{\dagger}\left(F_{z}\right)_{r s} \zeta_{s}=0\right)$ the anomalous vertex vectors are

$$
\begin{aligned}
& { }_{p}^{0} \underline{\Lambda}_{\alpha}=\left(\begin{array}{c}
\Lambda_{0 \alpha}^{++} \\
\Lambda_{0 \alpha}^{00} \\
\Lambda_{0 \alpha}^{--}
\end{array}\right), \quad{ }_{p}^{0} \underline{\Lambda}^{\alpha}=\left(\begin{array}{c}
\Lambda_{b \alpha}^{0 \alpha} \\
\Lambda_{00}^{0 \alpha} \\
\Lambda_{--}^{0 \alpha}
\end{array}\right), \\
& { }_{p}^{+} \underline{\Lambda}_{1}=\left(\begin{array}{c}
\Lambda_{-, 1}^{0+} \\
\Lambda_{-, 1}^{-0}
\end{array}\right), \quad \quad{ }_{p}^{+} \underline{\Lambda}^{1}=\left(\begin{array}{c}
\Lambda_{+0}^{-, 1} \\
\Lambda_{0-}^{-, 1}
\end{array}\right), \quad{ }_{p}^{+} \underline{\Lambda}_{-1}=\left(\begin{array}{c}
\Lambda_{+,-1}^{0+} \\
\Lambda_{+,-1}^{-0}
\end{array}\right), \quad{ }_{p}^{+} \underline{\Lambda}^{-1}=\left(\begin{array}{c}
\Lambda_{+0}^{+,-1} \\
\Lambda_{0-}^{+,-1}
\end{array}\right), \\
& { }_{p}^{-} \underline{\Lambda}_{1}=\left(\begin{array}{c}
\Lambda_{+, 1}^{+0} \\
\Lambda_{+, 1}^{0-}
\end{array}\right), \quad \bar{p} \underline{\Lambda}^{1}=\left(\begin{array}{c}
\Lambda_{0+}^{+, 1} \\
\Lambda_{-0}^{+, 1}
\end{array}\right), \quad \bar{p}_{-1}=\left(\begin{array}{c}
\Lambda_{-,-1}^{+0} \\
\Lambda_{-,-1}^{0-}
\end{array}\right), \quad \bar{p}^{-} \underline{\Lambda}^{-1}=\left(\begin{array}{c}
\Lambda_{0+-,-1}^{-,} \\
\Lambda_{-0}^{-,-1}
\end{array}\right) .
\end{aligned}
$$

For the ferromagnetic case (where $c=\zeta_{r}^{\dagger}\left(F_{z}\right)_{r s} \zeta_{s}=1$ ) it is easy to verify that spin conservation forbids any spin index for ${ }^{+} \underline{\Lambda}_{-1}$ and for ${ }^{-} \underline{\Lambda}_{1}$. These later vectors can be taken as zero.

$$
\begin{aligned}
& { }_{f}^{0} \underline{\Lambda}_{\alpha}=\left(\begin{array}{c}
\Lambda_{+\alpha}^{++} \\
\Lambda_{+\alpha}^{00} \\
\Lambda_{+\alpha}^{-\alpha}
\end{array}\right), \quad{ }_{f}^{0} \underline{\Lambda}^{\alpha}=\left(\begin{array}{c}
\Lambda_{++}^{+\alpha} \\
\Lambda_{00}^{+\alpha} \\
\Lambda_{--}^{+\alpha}
\end{array}\right), \\
& { }_{f}^{+} \underline{\Lambda}_{1}=\left(\begin{array}{c}
\Lambda_{0,1}^{0+} \\
\Lambda_{0,1}^{-0}
\end{array}\right), \quad \quad{ }_{f}^{+} \underline{\Lambda}^{1}=\left(\begin{array}{c}
\Lambda_{+0}^{0,1} \\
\Lambda_{0-1}^{0,1}
\end{array}\right), \quad{ }_{f}^{+} \underline{\Lambda}_{-1}=\underline{0}, \quad{ }_{f}^{+} \underline{\Lambda}^{-1}=\underline{0}, \\
& \bar{f}^{-} \underline{\Lambda}_{1}=\underline{0}, \quad-\quad \underline{\Lambda}^{1}=\underline{0}, \quad-\underline{\Lambda}_{-1}=\left(\begin{array}{c}
\Lambda_{0,-1}^{+0} \\
\Lambda_{0,-1}^{0-}
\end{array}\right), \quad \bar{f}^{-} \underline{\Lambda}^{-1}=\left(\begin{array}{c}
\Lambda_{0,-1}^{0,-1} \\
\Lambda_{-0}^{0,-1}
\end{array}\right) .
\end{aligned}
$$

for the ferromagnetic case. The symmetry relations of Eq. (25) hold for the proper and irreducible parts as well, which is equivalent to:

$$
\begin{gathered}
{ }^{0} \underline{\Lambda}^{\alpha}\left(\mathbf{k}, i \omega_{n}\right)={ }^{0} \underline{\Lambda}^{\alpha}\left(k, i \omega_{n}\right)={ }^{0} \underline{\Lambda}_{\alpha}\left(k, i \omega_{n}\right)={ }^{0} \underline{\Lambda}_{-\alpha}\left(k,-i \omega_{n}\right)={ }^{0} \underline{\Lambda}^{-\alpha}\left(k,-i \omega_{n}\right), \\
{ }^{+} \underline{\Lambda}^{\alpha}\left(\mathbf{k}, i \omega_{n}\right)={ }^{+} \underline{\Lambda}^{\alpha}\left(k, i \omega_{n}\right)={ }^{+} \underline{\Lambda}_{\alpha}\left(k, i \omega_{n}\right)={ }^{-} \underline{\Lambda}_{-\alpha}\left(k,-i \omega_{n}\right)={ }^{-} \underline{\Lambda}^{-\alpha}\left(k,-i \omega_{n}\right) .
\end{gathered}
$$

\section{E. Dielectric functions}

With the definition of the

$$
\begin{aligned}
& \varepsilon_{c d}^{s r}\left(\mathbf{k}, i \omega_{n}\right)=\delta_{d}^{s} \delta_{c}^{r}-\Pi_{a b}^{s r}\left(\mathbf{k}, i \omega_{n}\right) V_{c d}^{b a}, \\
& { }^{0}\left(\mathbf{k}, i \omega_{n}\right)={ }^{0} \underline{\underline{ }}-{ }^{0} \underline{\underline{\underline{\Pi}}}\left(\mathbf{k}, i \omega_{n}\right) \cdot{ }^{0} \underline{\underline{\underline{C}}}, \\
& { }^{ \pm} \underline{\underline{\varepsilon}}\left(\mathbf{k}, i \omega_{n}\right)={ }^{ \pm} \underline{\underline{1}}-{ }^{ \pm} \underline{\underline{\Pi}}\left(\mathbf{k}, i \omega_{n}\right) \cdot{ }^{ \pm} \underline{\underline{C}}
\end{aligned}
$$

dielectric functions Eqs. (29), (41), (43) and (45) can be rewritten as

$$
\begin{aligned}
& \varepsilon_{c d}^{s r}\left(\mathbf{k}, i \omega_{n}\right) D_{r^{\prime} s^{\prime}}^{d c}\left(\mathbf{k}, i \omega_{n}\right)=\hbar \Pi_{r^{\prime} s^{\prime}}^{s r}\left(\mathbf{k}, i \omega_{n}\right), \\
& { }^{0} \underline{\underline{\varepsilon}}\left(\mathbf{k}, i \omega_{n}\right) \cdot{ }^{0} \underline{\underline{D}}\left(\mathbf{k}, i \omega_{n}\right)=\hbar{ }^{0} \underline{\underline{ }}\left(\mathbf{k}, i \omega_{n}\right), \\
& { }^{ \pm} \underline{\underline{\varepsilon}}\left(\mathbf{k}, i \omega_{n}\right) \cdot{ }^{ \pm} \underline{\underline{D}}\left(\mathbf{k}, i \omega_{n}\right)=\hbar{ }^{ \pm} \underline{\underline{\Pi}}\left(\mathbf{k}, i \omega_{n}\right) .
\end{aligned}
$$

\section{F. Interaction propagator}

With the use of the proper parts of the density correlation functions one can define an interaction propagator:

$$
\mathcal{W}_{r^{\prime} s^{\prime}}^{r s}=V_{r^{\prime} s^{\prime}}^{r s}+\mathcal{W}_{a b}^{r s} \Pi_{c d}^{b a} V_{r^{\prime} s^{\prime}}^{d c}
$$


which can be symbolized as depicted in Fig. 11. Using the dielectric functions one can get

$$
\mathcal{W}_{a b}^{r s} \varepsilon_{r^{\prime} s^{\prime}}^{b a}=V_{r^{\prime} s^{\prime}}^{r s},
$$

This equation also splits to parts according to the spin transfer.

$$
\begin{aligned}
{ }^{0} \underline{\underline{\mathcal{W}}}{ }^{0} \underline{\underline{\varepsilon}}={ }^{0} \underline{\underline{C}} \\
{ }^{ \pm} \underline{\underline{\underline{\mathcal{W}}}}{ }^{ \pm} \underline{\underline{\varepsilon}}={ }^{ \pm} \underline{\underline{C}} \\
\mathcal{W}_{-+}^{+-}=\mathcal{W}_{+-}^{-+}=0 .
\end{aligned}
$$

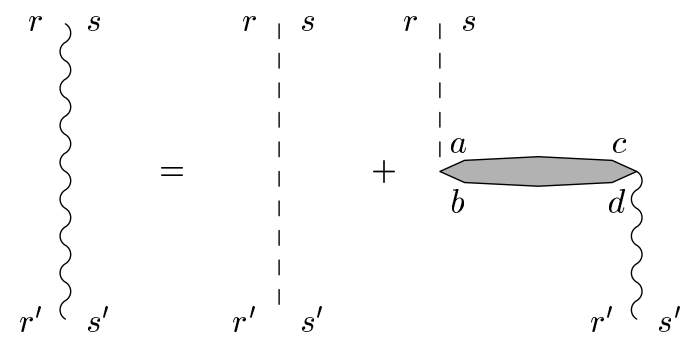

FIG. 11. The Feynman graph of the interaction propagator

\section{G. Proper Green's function and irreducible polarization part}

With a simple substitution one can verify that both Eq. (28) and Eq. (55a) have the following partition property. If one decomposes the self-energy and the proper part of (144) into two

$$
\begin{aligned}
\Sigma_{\gamma \delta}^{r s} & =\Sigma_{\gamma \delta}^{(1) r s}+\Sigma_{\gamma \delta}^{(2) r s}, \\
\Pi_{r^{\prime} s^{\prime}}^{s r} & =\Pi_{r^{\prime} s^{\prime}}^{(1) s r}+\Pi_{r^{\prime} s^{\prime}}^{(2) s r},
\end{aligned}
$$

then one can define propagators corresponding e.g. to the first part of these quantities

$$
\begin{aligned}
& \mathcal{G}_{\gamma \delta}^{(1) r s}=\mathcal{G}_{(0) \gamma \delta}^{r s}+\mathcal{G}_{(0) \gamma \sigma}^{r r^{\prime}} \Sigma_{\sigma \rho}^{(1) r^{\prime} s^{\prime}} \mathcal{G}_{\rho \delta}^{(1) s^{\prime} s}, \\
& \mathcal{W}_{r^{\prime} s^{\prime}}^{(1) r s}=V_{r^{\prime} s^{\prime}}^{r s}+\mathcal{W}_{a b}^{(1) r s} \Pi_{c d}^{(1) b a} V_{r^{\prime} s^{\prime}}^{d c}
\end{aligned}
$$

in such a way that

$$
\begin{aligned}
& \mathcal{G}_{\gamma \delta}^{r s}=\mathcal{G}_{\gamma \delta}^{(1) r s}+\mathcal{G}_{\gamma \sigma}^{(1) r r^{\prime}} \Sigma_{\sigma \rho}^{(2) r^{\prime} s^{\prime}} \mathcal{G}_{\rho \delta}^{s^{\prime} s}, \\
& \mathcal{W}_{r^{\prime} s^{\prime}}^{r s}=\mathcal{W}_{r^{\prime} s^{\prime}}^{(1) r s}+\mathcal{W}_{a b}^{r s} \Pi_{c d}^{(2) b a} \mathcal{W}_{r^{\prime} s^{\prime}}^{(1) d c}
\end{aligned}
$$

is fulfilled at the same time. Specially if one decomposes the self-energies by (30a) and the proper graphs by (30b) it defines the proper Green's functions

$$
\widetilde{\mathcal{G}}_{\gamma \delta}^{r s}=\mathcal{G}_{(0) \gamma \delta}^{r s}+\mathcal{G}_{(0) \gamma \sigma}^{r r^{\prime}} \widetilde{\Sigma}_{\sigma \rho}^{r^{\prime} s^{\prime}} \widetilde{\mathcal{G}}_{\rho \delta}^{s^{\prime} s}
$$

and an effective potential

$$
W_{r^{\prime} s^{\prime}}^{r s}=V_{r^{\prime} s^{\prime}}^{r s}+W_{a b}^{r s} \Pi_{c d}^{(r) b a} V_{r^{\prime} s^{\prime}}^{d c}
$$

such a way that

$$
\begin{aligned}
\mathcal{G}_{\gamma \delta}^{r s} & =\widetilde{\mathcal{G}}_{\gamma \delta}^{r s}+\widetilde{\mathcal{G}}_{\gamma \sigma}^{r r^{\prime}} M_{\sigma \rho}^{r^{\prime} s^{\prime}} \mathcal{G}_{\rho \delta}^{s^{\prime} s}, \\
\mathcal{W}_{r^{\prime} s^{\prime}}^{r s} & =W_{r^{\prime} s^{\prime}}^{r s}+\mathcal{W}_{a b}^{r s} \Pi_{c d}^{(s) b a} W_{r^{\prime} s^{\prime}}^{d c}
\end{aligned}
$$


is fulfilled. The earlier discussed symmetry properties hold for these decomposed parts as well (since these are defined by a class of graphs) and as consequence, Eqs. (62), (64a) and Eqs. (63), (64b) split to matrix equations in the same way as Eq. (28) and Eq. (29) did. It is convenient to define the regular part of the dielectric functions with the

$$
\begin{gathered}
\varepsilon_{c d}^{(r) s r}=\delta_{d}^{s} \delta_{c}^{r}-\Pi_{a b}^{(r) s r} V_{c d}^{b a} \\
{ }^{0} \underline{\underline{\varepsilon}}^{(r)}={ }^{0} \underline{\underline{1}}-{ }^{0} \underline{\underline{\Pi}}^{(r)} \cdot{ }^{0} \underline{\underline{C}} \\
{ }^{ \pm}{ }^{\underline{\underline{\varepsilon}}} \\
(r)
\end{gathered}
$$

equations which can be used to express the effective potential as:

$$
\begin{aligned}
{ }^{0} \underline{\underline{W}}{ }^{0} \underline{\underline{\varepsilon}}^{(r)} & ={ }^{0} \underline{\underline{C}}, \\
{ }^{ \pm} \underline{\underline{W}}{ }^{ \pm} \underline{\underline{\varepsilon}} & ={ }^{ \pm} \underline{\underline{C}}, \\
W_{-+}^{+-} & =W_{+-}^{-+}=0 .
\end{aligned}
$$

\section{H. Improper self-energy and singular polarization}

With the use of the irreducible and proper anomalous vertex functions and the effective potential one can construct the improper self energies (as seen in Fig. 12.):

$$
\begin{aligned}
\hbar M_{\alpha \beta}^{a b}\left(\mathbf{k}, i \omega_{n}\right) & =\widetilde{\Lambda}_{c d}^{a \alpha}\left(\mathbf{k}, i \omega_{n}\right) W_{e f}^{d c}\left(\mathbf{k}, i \omega_{n}\right) \widetilde{\Lambda}_{b \beta}^{f e}\left(\mathbf{k}, i \omega_{n}\right), \\
\hbar{ }^{0} M_{\alpha \beta} & ={ }^{0} \underline{\widetilde{\Lambda}}^{\alpha}{ }^{0} \underline{\underline{W}}{ }^{0} \widetilde{\widetilde{\Lambda}}_{\beta}={ }^{0} \underline{\widetilde{\Lambda}}^{\alpha}{ }^{0} \underline{\underline{C}}^{0}{ }^{0} \underline{\varepsilon}^{(r)^{-1}}{ }^{0} \underline{\widetilde{\Lambda}}_{\beta}, \\
\hbar{ }^{ \pm} M_{\alpha \beta} & ={ }^{ \pm} \underline{\widetilde{\Lambda}}^{\alpha}{ }^{ \pm} \underline{\underline{W}}{ }^{ \pm} \underline{\widetilde{\Lambda}}_{\beta}={ }^{ \pm} \underline{\widetilde{\Lambda}}^{\alpha}{ }^{ \pm} \underline{\underline{C}}^{ \pm} \underline{\underline{\varepsilon}}^{(r)^{-1}}{ }^{ \pm} \underline{\widetilde{\Lambda}}_{\beta} .
\end{aligned}
$$

It is easy to verify that $W_{+-}^{-+}=0$ which means that for the ferromagnetic case the improper self-energy in the \pm 2 (quadrupolar) spin transfer mode

$$
{ }^{ \pm Q} M=0 .
$$

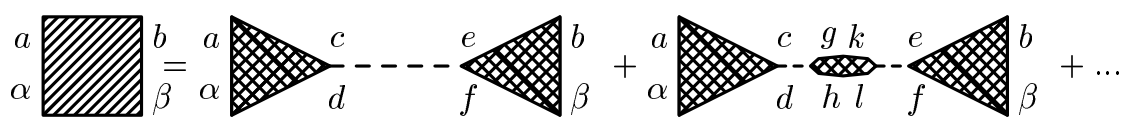

FIG. 12. The structure of the perturbation series of the improper self-energy

The singular polarization can be expressed as well with the use of the proper Green's functions and the proper anomalous vertices as seen in Fig. 13. and is of the form:

$$
\begin{aligned}
\hbar \Pi_{r^{\prime} s^{\prime}}^{(s) s r}\left(\mathbf{k}, i \omega_{n}\right) & =\widetilde{\Lambda}_{a \alpha}^{s r}\left(\mathbf{k}, i \omega_{n}\right) \widetilde{\mathcal{G}}_{\alpha \beta}^{a b}\left(\mathbf{k}, i \omega_{n}\right) \widetilde{\Lambda}_{r^{\prime} s^{\prime}}^{b \beta}\left(\mathbf{k}, i \omega_{n}\right), \\
\hbar^{0} \underline{\underline{\Pi}}^{(s)} & ={ }^{0} \widetilde{\mathcal{G}}_{\alpha \beta}{ }^{0} \underline{\widetilde{\Lambda}}_{\alpha} \circ{ }^{0} \underline{\widetilde{\Lambda}}^{\beta}, \\
\hbar^{ \pm} \underline{\underline{\Pi}}^{(s)} & ={ }^{ \pm} \widetilde{\mathcal{G}}_{\alpha \beta}{ }^{ \pm} \underline{\widetilde{\Lambda}}_{\alpha} \circ{ }^{ \pm} \underline{\widetilde{\Lambda}}^{\beta},
\end{aligned}
$$

where the circle denotes the diadic product operation. For the polar case the singular polarization in the \pm 2 spin transfer mode is equal to zero since there is no corresponding proper Green's function. For the ferromagnetic case e.g. for the +2 spin transfer mode the singular polarization is:

$$
\hbar_{f} \Pi_{+-}^{(s)-+}={ }_{f} \widetilde{\Lambda}_{-, 1}^{-+}{ }_{f} \widetilde{\mathcal{G}}_{11}^{--}{ }_{f} \widetilde{\Lambda}_{+-}^{-, 1} .
$$




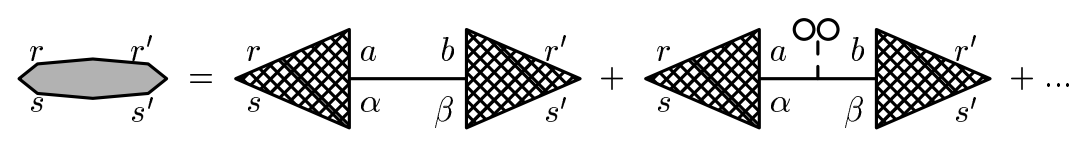

FIG. 13. The diagrammatic structure of the singular polarization

As for the scalar case [31,30] one can see intermediate states corresponding to collective excitations in the perturbation series of the one-particle propagators and at the same time one can identify one-particle intermediate states in the perturbation series of the correlation functions of the collective modes. However there is a great difference between the scalar and the spinor models. The rotational invariance of the interaction potential results in that $V_{+-}^{-+}=V_{-+}^{+-}=0$ which leads to the proper nature of the quadrupolar spin density correlation functions. This combined with the fact that in the polar case there is no anomalous vertex with \pm 2 spin transfer results in the prohibition of the coupling to any of the one-particle correlation functions in this phase.

\section{Couplings amongst the correlation functions}

With the help of the quantities discussed in the previous subsection the properties of the density correlation functions can be further investigated. For this reason let us introduce the following vectors: $\underline{\xi}_{1}=(1,1,1)^{\dagger} / \sqrt{3}$, $\underline{\xi}_{2}=(1,0,-1)^{\dagger} / \sqrt{2}, \underline{\xi}_{3}=(1,-2,1)^{\dagger} / \sqrt{6}$ for the zero spin transfer mode and $\underline{\chi}_{1}=(1,1)^{\dagger} / \sqrt{2}$ and $\underline{\chi}_{2}=(1,-1)^{\dagger} / \sqrt{2}$ for the \pm 1 spin transfer mode. It is easy to verify that $\left\{\underline{\xi}_{1}, \underline{\xi}_{2}, \underline{\xi}_{3}\right\}$ and $\left\{\underline{\chi}_{1}, \underline{\chi}_{2}\right\}$ are orthogonal and normalized basis sets in the linear vector spaces with dimension three and two, respectively. The density correlation functions (14a), 14b), 14c) and (14d) can be expressed as follows:

$$
\begin{gathered}
D_{n n}=3\left(\underline{\xi}_{1}^{\dagger}{ }^{0} \underline{\underline{D}} \underline{\xi}_{1}\right), \quad D_{z z}=2\left(\underline{\xi}_{2}^{\dagger}{ }^{0} \underline{\underline{D}} \underline{\xi}_{2}\right), \quad D_{n z}=\sqrt{6}\left(\underline{\xi}_{1}^{\dagger}{ }^{0} \underline{\underline{D}} \underline{\xi}_{2}\right) \\
D_{++}=4\left(\underline{\chi}_{1}^{\dagger}{ }^{+} \underline{\underline{D}} \underline{\chi}_{1}\right), \quad D_{--}=4\left(\underline{\chi}_{1}^{\dagger} \underline{\underline{D}} \underline{\chi}_{1}\right) .
\end{gathered}
$$

Since ${ }^{0} \underline{\underline{D}}$ is a symmetric matrix $D_{n z}=D_{z n}$ holds.

Multiplying Eq. (43) with $2 \underline{\chi}_{1}$ from both sides and using that ${ }^{+} \underline{\underline{C}}=2 c_{s} \underline{\chi}_{1} \circ \underline{\chi}_{1}$ one obtains

$$
D_{++}=\hbar \Pi_{++}+\frac{c_{s}}{2} \Pi_{++} D_{++}
$$

where $\Pi_{++}=4 \underline{\chi}_{1}^{\dagger+} \underline{\underline{\Pi}} \chi_{1}$. A similar equation can be derived to $D_{--}$. There are both regular and singular contributions to these propagators.

One can get a closed system of equations for the zero spin transfer correlation functions of Eq. (14a), Eq. (14b) and Eq. (140). To this end first we multiply Eq. 41 with $\sqrt{3} \underline{\xi}_{1}$ from both sides. We further use that ${ }^{0} \underline{\underline{C}}=$ $3 c_{n} \underline{\xi}_{1} \circ \underline{\xi}_{1}+2 c_{s} \underline{\xi}_{2} \circ \underline{\xi}_{2}$. The resulting equations sound as

$$
D_{n n}=\hbar \Pi_{n n}+c_{n} \Pi_{n n} D_{n n}+c_{s} \Pi_{n z} D_{n z}
$$

where $\Pi_{n n}=3 \underline{\xi}_{1}{ }^{0} \underline{\underline{\Pi}} \xi_{1}$ and $\Pi_{n z}=\sqrt{6} \underline{\xi}_{1}{ }^{0} \underline{\underline{\Pi}}_{2}$. Multiplying (41) with $\sqrt{2} \underline{\xi}_{2}$ from both sides one gets

$$
D_{z z}=\hbar \Pi_{z z}+c_{n} \Pi_{n z} D_{n z}+c_{s} \Pi_{z z} D_{z z}
$$

with $\Pi_{z z}=2 \underline{\xi}_{2}{ }^{0} \underline{\underline{\Pi}}_{2}$. The third equation can be obtained by multiplying Eq. (41) with $\sqrt{3} \underline{\xi}_{1}$ from the left hand side and with $\sqrt{2} \underline{\xi}_{2}$ from the right hand side which leads to

$$
D_{n z}=\hbar \Pi_{n z}+c_{n} \Pi_{n n} D_{n z}+c_{s} \Pi_{n z} D_{z z}
$$

These coupled equations can be solved for the correlation functions. The polarization part $\Pi_{n z}$ written out in detail reads as

$$
\Pi_{n z}=\Pi_{++}^{++}-\Pi_{--}^{--}+\Pi_{++}^{00}-\Pi_{--}^{00}
$$


It is obviously zero if the system is invariant under spin reflection, i.e., when those matrix elements coincide which can be get from each other by reverting a + to a - and vice versa. This condition is fulfilled for both cases in the symmetric phase, moreover for the polar case it even holds throughout the condensed phase. With $\Pi_{n z}=0$ Eqs. (72a) and (72b) are independent, giving the solutions

$$
\begin{aligned}
& D_{n n}=\frac{\hbar \Pi_{n n}}{1-c_{n} \Pi_{n n}} \\
& D_{n z}=0 \\
& D_{z z}=\frac{\hbar \Pi_{z z}}{1-c_{s} \Pi_{z z}}
\end{aligned}
$$

and leading to two separate excitation spectra. The singular part of $\Pi_{z z}$ can be cast with Eq. (68b) to the form

$$
\hbar \Pi_{z z}^{(s)}=2\left(\underline{\xi}_{2}{ }^{0} \underline{\widetilde{\Lambda}}_{\alpha}\right)\left({ }^{0} \underline{\widetilde{\Lambda}}^{\beta} \underline{\xi}_{2}\right){ }^{0} \widetilde{\mathcal{G}}_{\alpha \beta}
$$

which is zero if spin reflection symmetry is present, since the anomalous vertex vector ${ }_{p}^{0} \underline{\widetilde{\Lambda}}_{\alpha}$ is orthogonal to $\underline{\xi}_{2}$ in this case.

In the ferromagnetic phase the spectra of collective excitations corresponding to spin density waves and density waves with $n=0$ are coupled. Above the critical temperature this coupling vanishes and the two modes will be independent. In the polar phase spin density fluctuations will always be independent of the particle density fluctuations, furthermore $\Pi_{z z}^{(s)}=0$ which means that the spin density correlation function $74 \mathrm{~d}$ ) has only regular contribution.

In the polar phase further analysis leads to results important for the following. First note that, when the system exhibits the spin reflection symmetry, ${ }^{0} \underline{\underline{D}}$ and ${ }^{0} \underline{\underline{ }}$ have only 4 independent elements (instead of 6 characteristic to a symmetric matrix). These independent elements are $\Pi_{++}^{++}=\Pi_{--}^{--}, \Pi_{++}^{--}=\Pi_{--}^{++}, \Pi_{++}^{00}=\Pi_{00}^{++}=\Pi_{--}^{00}=\Pi_{00}^{--}$and $\Pi_{00}^{00}$. Second, these can be cast into a more practical form with the help of the orthogonal matrix

$$
\underline{\underline{O}}=\left[\begin{array}{ccc}
\frac{\sqrt{3}}{3} & \frac{\sqrt{3}}{3} & \frac{\sqrt{3}}{3} \\
\frac{\sqrt{2}}{2} & 0 & -\frac{\sqrt{2}}{2} \\
\frac{\sqrt{6}}{6} & -\frac{\sqrt{6}}{3} & \frac{\sqrt{6}}{6}
\end{array}\right]
$$

Its rows are built from the vectors $\left\{\underline{\xi}_{1}, \underline{\xi}_{2}, \underline{\xi}_{3}\right\}$. Performing the transformation leads to

$$
\begin{gathered}
\underline{\underline{C}}^{\prime}:={ }^{0} \underline{\underline{\mathcal{O}}} \cdot{ }^{0} \underline{\underline{C}} \cdot{ }^{0} \underline{\underline{\mathcal{O}}}^{T}=\left[\begin{array}{ccc}
3 c_{n} & 0 & 0 \\
0 & 2 c_{s} & 0 \\
0 & 0 & 0
\end{array}\right], \\
\underline{\underline{\Pi}}^{(x)^{\prime}}:={ }^{0} \underline{\underline{\mathcal{O}}} \cdot{ }^{0} \underline{\underline{\Pi}}^{(x)} \cdot{ }^{0} \underline{\underline{\mathcal{O}}}^{T}=\left[\begin{array}{ccc}
\frac{\Pi_{n n}^{(x)}}{3} & 0 & \frac{\Pi_{13}^{(x)}}{3 \sqrt{2}} \\
0 & \frac{\Pi_{z z}^{(x)}}{2} & 0 \\
\frac{\Pi_{13}^{(x)}}{3 \sqrt{2}} & 0 & \frac{\Pi_{33}^{(x)}}{6}
\end{array}\right],
\end{gathered}
$$

with $\Pi_{13}^{(x)}=3 \sqrt{2} \underline{\xi}_{1}{ }^{0} \underline{\Pi}^{(x)} \underline{\xi}_{3}$ and $\Pi_{33}^{(x)}=6 \underline{\xi}_{3}{ }^{0} \underline{\underline{\Pi}}^{(x)} \underline{\xi}_{3}$, where $x$ can be $r$ or $s$ corresponding to the regular or the singular parts, respectively. Furthermore, according to $(75) \Pi_{z z}^{(s)}=0$.

The total polarization matrix therefore becomes

$$
{ }^{0} \underline{\underline{ }}^{\prime}=\left[\begin{array}{ccc}
\frac{\Pi_{n n}^{(r)}+\Pi_{n n}^{(s)}}{3} & 0 & \frac{\Pi_{13}^{(r)}+\Pi_{13}^{(s)}}{3 \sqrt{2}} \\
0 & \frac{\Pi_{z z}^{(r)}}{2} & 0 \\
\frac{\Pi_{13}^{(r)}+\Pi_{13}^{(s)}}{3 \sqrt{2}} & 0 & \frac{\Pi_{33}^{(r)}+\Pi_{33}^{(s)}}{6}
\end{array}\right] .
$$

Using Eqs. (77a) and (78) the dielectric function (52b), after the transformation, can be cast into the form

$$
{ }^{0} \underline{\underline{\varepsilon}}^{\prime}=\left[\begin{array}{ccc}
1-c_{n}\left(\Pi_{n n}^{(r)}+\Pi_{n n}^{(s)}\right) & 0 & 0 \\
0 & 1-c_{s} \Pi_{z z}^{(r)} & 0 \\
-\frac{c_{n}}{\sqrt{2}}\left(\Pi_{13}^{(r)}+\Pi_{13}^{(s)}\right) & 0 & 1
\end{array}\right]
$$


We arrive at the important result, that the determinant of the dielectric function, which is invariant under such transformations, reads as

$$
\operatorname{det}^{0} \underline{\underline{\varepsilon}}=\operatorname{det}^{0} \underline{\underline{\varepsilon^{\prime}}}=\left[1-c_{n}\left(\Pi_{n n}^{(r)}+\Pi_{n n}^{(s)}\right)\right]\left(1-c_{s} \Pi_{z z}^{(r)}\right)
$$

This determinant factorize into two in agreement with the separation of the density and spin density fluctuations. Furthermore it shares one factor with its regular counterpart since

$$
\operatorname{det} \stackrel{\underline{\varepsilon}}{=}^{(r)}=\left(1-c_{n} \Pi_{n n}^{(r)}\right)\left(1-c_{s} \Pi_{z z}^{(r)}\right)
$$

which can be obtained by taking the determinant of Eq. (79) after setting all $\Pi^{(s)}=0$.

Another important consequence of the spin reflection symmetry is that the interaction propagator (55b) and its regular part (66a) has the same structure as the interaction matrix (77a):

$$
{ }^{0} \underline{\underline{\mathcal{W}}}^{\prime}=\left[\begin{array}{ccc}
3 \mathcal{C}_{n} & 0 & 0 \\
0 & 2 \mathcal{C}_{s} & 0 \\
0 & 0 & 0
\end{array}\right], \quad{ }^{0} \underline{\underline{W}}^{\prime}=\left[\begin{array}{ccc}
3 \mathcal{C}_{n}^{(r)} & 0 & 0 \\
0 & 2 \mathcal{C}_{s}^{(r)} & 0 \\
0 & 0 & 0
\end{array}\right]
$$

with

$$
\begin{aligned}
\mathcal{C}_{n} & =\frac{c_{n}}{1-c_{n} \Pi_{n n}}, & \mathcal{C}_{n}^{(r)} & =\frac{c_{n}}{1-c_{n} \Pi_{n n}^{(r)}} \\
\mathcal{C}_{s} & =\frac{c_{s}}{1-c_{s} \Pi_{z z}}, & \mathcal{C}_{s}^{(r)} & =\frac{c_{s}}{1-c_{s} \Pi_{z z}^{(r)}}
\end{aligned}
$$

Those correlation functions which have singular polarization parts correspond to fluctuations belonging both to the condensate and to the noncondensate, while those which have no singular proper part only belong to the noncondensate (they can not couple to any of the one-particle modes). So in the polar phase the spin density fluctuations described by $D_{z z}$ (which are independent from the particle density fluctuations described by $D_{n n}$ ) belong only to the noncondensate. The singular polarization parts of the quadrupolar modes are also zero (since there is no corresponding Green's function and the anomalous vertices of the \pm 2 spin transfer are also zero) meaning that these type of fluctuations also belong only to the noncondensate. The proper parts $\Pi_{n n}, \Pi_{++}$and $\Pi_{--}$have singular contributions too with intermediate

one-particle states ${ }^{0} \widetilde{\mathcal{G}}_{\alpha \beta}, \quad+\widetilde{\mathcal{G}}_{\alpha \beta}$ and $-\widetilde{\mathcal{G}}_{\alpha \beta}$, respectively. These modes are coupled to the condensate. In the ferromagnetic phase $D_{z z}$ and $D_{n n}$ are coupled (their cross correlation $D_{n z}$ do not vanish either), their denominators are common. All of their proper parts have singular contributions resulting in their coupling to the one-particle excitations with ${ }^{0} \widetilde{\mathcal{G}}$. These fluctuations thus belong both to the condensate and noncondensate. The correlation functions of the spin waves (both the \pm 1 and \pm 2 spin transfer modes) $D_{++}, D_{--}, D_{++}^{Q}$ and $D_{--}^{Q}$ have singular proper parts connecting them with the propagators ${ }^{+} \widetilde{\mathcal{G}},-\widetilde{\mathcal{G}}, Q \widetilde{\mathcal{G}}$ and $-Q \widetilde{\mathcal{G}}$, respectively. Note, that there are no improper self-energies for the quadrupolar spin transfer modes, consequently their proper Green's functions are the same as the full propagators of these two modes.

\section{J. Coupling between the Green's functions and the correlation functions and the spectra of excitations}

A) Let us first consider the modes with $n=0,+,-$. With the decomposition (30a) of the self-energies and with a straightforward calculation starting from Eq. (37) one arrives at the expression

$$
{ }^{n} \mathcal{G}_{\alpha \gamma} \equiv \frac{{ }^{n} N_{\alpha \gamma}}{{ }^{n} \Delta}=\frac{{ }^{n} \widetilde{N}_{\alpha \gamma}+\alpha \gamma{ }^{n} M_{-\gamma,-\alpha}}{{ }^{n} \widetilde{\Delta}-{ }^{n} \widetilde{N}_{\sigma \tau}{ }^{n} M_{\tau \sigma}-\operatorname{det}{ }^{n} M}
$$

both for the polar and ferromagnetic phases. Here we introduced the

$$
\begin{aligned}
{ }^{n} \widetilde{N}_{\alpha \gamma} & =\delta_{\alpha \gamma}\left(\alpha i \omega_{n}+\hbar^{-1} \epsilon_{\mathbf{k}}\right)+\alpha \gamma{ }^{n} \widetilde{\Sigma}_{-\gamma,-\alpha}, \\
{ }^{n} \widetilde{\Delta} & =\left(i \omega_{n}-\hbar^{-1} \epsilon_{\mathbf{k}}-{ }^{n} \widetilde{\Sigma}_{11}\right)\left(i \omega_{n}+\hbar^{-1} \epsilon_{\mathbf{k}}+{ }^{n} \widetilde{\Sigma}_{-1,-1}\right)+{ }^{n} \widetilde{\Sigma}_{-1,1}{ }^{n} \widetilde{\Sigma}_{1,-1}
\end{aligned}
$$


quantities, with ${ }^{n} \widetilde{\mathcal{G}}_{\alpha \gamma}={ }^{n} \widetilde{N}_{\alpha \gamma} /{ }^{n} \widetilde{\Delta}$ and $\operatorname{det}{ }^{n} M={ }^{n} M_{11}{ }^{n} M_{-1,-1}-{ }^{n} M_{1,-1}{ }^{n} M_{-1,1}$. With the help of Eqs. (53b) and (53c) the density correlation functions with $n=0,+,-$ can be brought to the form

$$
{ }^{n} \underline{\underline{D}}=\hbar \frac{{ }^{n} \underline{\underline{\underline{E}}}}{\operatorname{det}^{n} \underline{\underline{\underline{\underline{ }}}}}
$$

by writing

$$
{ }^{n} \underline{\underline{E}}=\operatorname{det}\left({ }^{n} \underline{\underline{\varepsilon}}\right){ }^{n} \underline{\underline{\varepsilon}}^{-1} .
$$

An important relationship can be shown in the following way. First applying the decomposition ${ }^{n} \underline{\underline{\varepsilon}}={ }^{n} \underline{\underline{\varepsilon}}^{(r)}+{ }^{n} \underline{\underline{\varepsilon}}^{(s)}$, with ${ }^{n} \underline{\underline{\varepsilon}}^{(s)}=-{ }^{n} \underline{\underline{\Pi}}^{(s)}{ }^{n} \underline{\underline{C}}$, to $\operatorname{det}^{n} \underline{\underline{\varepsilon}}$ leads to

$$
\operatorname{det}^{n} \underline{\underline{\varepsilon}}=\operatorname{det}\left({ }^{n} \underline{\underline{\varepsilon}}^{(r)}\right) \operatorname{det}\left(1-{ }^{n} \underline{\underline{\Pi}}^{(s)}{ }^{n} \underline{\underline{W}}\right) \text {. }
$$

With the help of Eqs. (68b) and (68d) and a straightforward but rather lengthy algebraic manipulation one can find that

$$
\begin{aligned}
\operatorname{det}\left(1-{ }^{n} \underline{\underline{M}}^{(s)}{ }^{n} \underline{\underline{W}}\right)=\operatorname{det}\left[\underline{\underline{1}}-\hbar^{-1}{ }^{n} \widetilde{\mathcal{G}}_{\alpha \beta}{ }^{n} \underline{\widetilde{\Lambda}}_{\alpha} \circ{ }^{n} \underline{\widetilde{\Lambda}}^{\beta}{ }^{n} \underline{\underline{W}}\right]=1-\hbar^{-1}{ }^{n} \widetilde{\mathcal{G}}_{\alpha \beta}{ }^{n} \underline{\underline{\Lambda}}_{\alpha}{ }^{n} \underline{\underline{W}}{ }^{n} \underline{\underline{\Lambda}}^{\beta} \\
\\
+\hbar^{-1} \operatorname{det}\left({ }^{n} \widetilde{\mathcal{G}}\right) \operatorname{det}\left({ }^{n} \underline{\widetilde{\Lambda}}_{\alpha}{ }^{n} \underline{\underline{W}}^{n} \underline{\widetilde{\Lambda}}^{\beta}\right) .
\end{aligned}
$$

Inserting it to Eq. (84) and using Eqs. (67b) and $(67 \mathrm{c})$ and that $\operatorname{det}{ }^{n} \widetilde{\mathcal{G}}=-{ }^{n} \widetilde{\Delta}^{-1}$ and comparing the result with the denominator of the Green's functions (22a) one can arrive at the basic connection

$$
{ }^{n} \widetilde{\Delta} \operatorname{det}^{n} \underline{\underline{\varepsilon}}={ }^{n} \Delta \operatorname{det}^{n} \underline{\underline{\varepsilon}}^{(r)} .
$$

Changing to retarded correlation functions, which is done in the usual way (by analytically continuing in frequency) [40], the elementary excitations of the system are determined by the poles of the corresponding correlation functions. The spectra of the one-particle excitations (quasiparticles) are given by the poles of the Green's functions, or equivalently by the equation

$$
{ }^{n} \Delta=0
$$

The spectra of collective excitations are determined by the poles of the density correlation functions, or equivalently by the equation

$$
\operatorname{det}^{n} \underline{\underline{\varepsilon}}=0 .
$$

Equation (86) shows that the Green's functions with spin transfer $n=0,+,-$ can be arranged to have the same denominator as the density correlation functions with the same spin transfer. This means that if det $^{n} \underline{\underline{\underline{\varepsilon}}}$ has a zero (the corresponding density correlation function, ${ }^{n} \underline{\underline{D}}$, has a pole) than ${ }^{n} \Delta$ must have a zero $\left({ }^{n} \mathcal{G}\right.$ must have a pole) there as well in general. The zero spin transfer mode in the polar case is an exception, where both the dielectric function and its regular part factorize and share some of their zeroes (see Eqs. (80a) and (80b)). In this case the zero of the left hand side coming from $\operatorname{det}^{0} \underline{\underline{\varepsilon}}$ satisfies the equation with the zero coming from $\operatorname{det}^{0} \underline{\underline{\varepsilon}}^{(r)}$ of the right hand side, instead of ${ }^{0} \Delta$. Hence, this zero do not appear amongst the poles of ${ }^{0} \mathcal{G}$. This further means that density correlation functions with 0 and \pm 1 spin transfer has common denominators with the Green's functions corresponding to the same spin transfer for both the polar and the ferromagnetic cases, except the $D_{z z}$ spin density correlation function in the polar case, which do not couple to any of the Green's functions.

B) Concerning the quadrupolar spin waves ( $n= \pm 2$ modes) let us consider first the ferromagnetic phase. Both the full Green's function and the correlation function are proper (see Eq. (67d), (46) and (47)) for such spin transfers. Consequently one can subtitute in Eq. (69) the full Green's function leading to

$$
D_{ \pm \pm}^{Q}=4\left(\Pi_{ \pm \mp}^{(r) \mp \pm}+\hbar^{-1}{ }_{f} \widetilde{\Lambda}_{-, \pm 1}^{\mp \pm} \mathcal{G}_{ \pm 1, \pm 1}^{--}{ }_{f} \widetilde{\Lambda}_{ \pm \mp}^{-, \pm 1}\right) .
$$

The one-particle excitations then appear also as collective ones.

In the polar case there is no Green's function with $n= \pm 2$, see Sec. IIID therefore these type of collective excitations, do not show up as one-particle elementary excitations.

Thus in the polar case the collective and one-particle excitation spectra do not match fully. There are excitation modes in this phase, which do not belong to the condensate. In the ferromagnetic case all of the collective excitation modes are connected to corresponding Green's functions, meaning that all types of density fluctuations are governed by condensate dynamics. 


\section{BOGOLIUBOV APPROXIMATION}

In this section we discuss the simplest approximation in the framework of the dielectric formalism, namely the Bogoliubov approximation. This approximation is a very low temperature calculation, it neglects all terms coming from the non-condensate density. This approximation was studied by other authors as well [14, 15 with other techniques. With the Green's function method one can give the form of the correlation functions as well not just the frequency of the modes.

The proper self-energies in the Bogoliubov approximation are

$$
\begin{aligned}
& \widetilde{\Sigma}_{\alpha \gamma}^{r s}=\hbar^{-1}\left[\left(\mu_{0}-\mu\right) \delta_{r s} \delta_{\alpha \gamma}+N_{0} \zeta_{r^{\prime}}^{\dagger} V_{r s}^{r^{\prime} s^{\prime}} \zeta_{s^{\prime}} \delta_{\alpha \gamma}\right], \\
& \widetilde{\Sigma}_{01}^{r}=\hbar^{-1} \sqrt{N_{0}}\left[(-\mu) \zeta_{r}+N_{0} \zeta_{s^{\prime}}^{\dagger} \zeta_{s}^{\dagger} V_{s r}^{s^{\prime} r^{\prime}} \zeta_{r^{\prime}}\right] .
\end{aligned}
$$

The corresponding Feynman-graphs can be seen in Fig. 14.

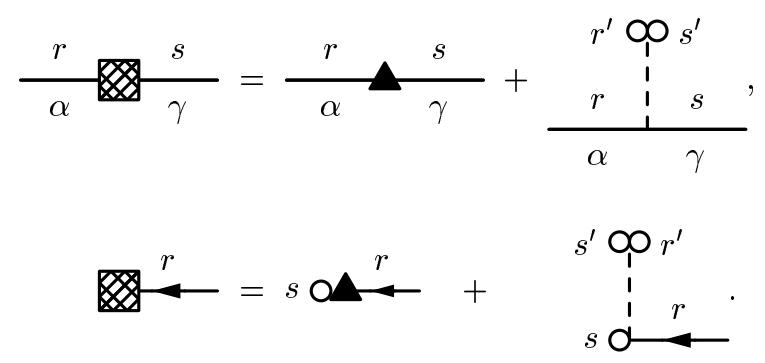

FIG. 14. The Feynman graphs of the proper self-energies and the tadpole diagrams in the Bogoliubov approximation

The regular polarization is zero in the Bogoliubov approximation and the proper anomalous vertex reads as

$$
\widetilde{\Lambda}_{a \alpha}^{s r}=\sqrt{N_{0}}\left[\delta_{r a} \delta_{\alpha,-1} \zeta_{s}+\delta_{s a} \delta_{\alpha, 1} \zeta_{r}^{\dagger}\right]
$$

which can be graphically represented as seen in Fig. 15.

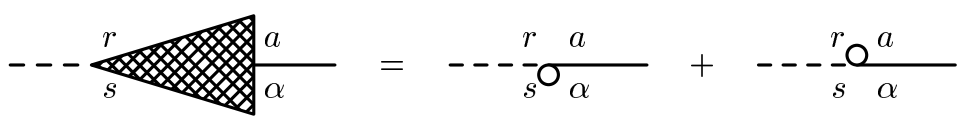

FIG. 15. The proper anomalous vertex of the Bogoliubov approximation

After calculating the proper Green's functions the improper self-energy and the singular polarization can be constructed from Eqs. (67a) and (68a). With their explicit form known the correlation functions can be expressed. These calculations have to be made separately for the polar and for the ferromagnetic cases.

\section{A. Polar case}

In the polar case $c_{s}>0$; the condensate spinor in this phase can be chosen to $\zeta_{r}=\delta_{r, 0}$. The chemical potential can be calculated using Eq. (34) which results in $\mu=N_{0} c_{n}$. Since the proper self-energy is zero (except the diagonal $\mu_{0}$ terms) the proper Green's functions will be:

$$
\widetilde{\mathcal{G}}_{\alpha \gamma}^{r s}\left(\mathbf{k}, i \omega_{n}\right)=\frac{\delta_{r s} \delta_{\alpha \gamma}}{\alpha i \omega_{n}-\hbar^{-1} e_{\mathbf{k}}} .
$$


This is similar to the free propagator of Eq. (27) but the $\mu_{0}$ parameter has been canceled out. The anomalous vertex vectors are:

$$
{ }_{p}^{0} \widetilde{\Lambda}_{\alpha}=\left(\begin{array}{c}
0 \\
\sqrt{N_{0}} \\
0
\end{array}\right), \quad{ }_{p}^{+} \underline{\Lambda}_{1}=\left(\begin{array}{c}
0 \\
\sqrt{N_{0}}
\end{array}\right), \quad{ }_{p}^{+} \underline{\Lambda}_{-1}=\left(\begin{array}{c}
\sqrt{N_{0}} \\
0
\end{array}\right)
$$

With the use of them the singular polarization matrices and the improper self-energies can be expressed using Eqs. (68) and (67). The singular polarization matrices are

$$
\underline{\underline{\Pi}}^{(s)}=\left[\begin{array}{ccc}
0 & 0 & 0 \\
0 & \Pi_{S} & 0 \\
0 & 0 & 0
\end{array}\right], \quad \quad \underline{\underline{\Pi}}^{(s)}=\left[\begin{array}{cc}
\Pi_{\mp} & 0 \\
0 & \Pi_{ \pm}
\end{array}\right],
$$

where $\Pi_{ \pm}=N_{0} /\left( \pm i \hbar \omega_{n}-e_{\mathbf{k}}\right)$ and $\Pi_{S}=\Pi_{+}+\Pi_{-}$were introduced. The improper self-energy matrices are:

$$
\underline{\underline{M}}=\hbar^{-1}\left[\begin{array}{cc}
N_{0} c_{n} & N_{0} c_{n} \\
N_{0} c_{n} & N_{0} c_{n}
\end{array}\right], \quad \quad \underline{\underline{M}}=\hbar^{-1}\left[\begin{array}{cc}
N_{0} c_{s} & N_{0} c_{s} \\
N_{0} c_{s} & N_{0} c_{s}
\end{array}\right] .
$$

Putting all together to Eq. (82a) the Green's functions will be

$$
\begin{aligned}
{ }^{0} \mathcal{G}_{\alpha \gamma} & =\frac{\delta_{\alpha \gamma}\left(\alpha i \omega_{n}+\hbar^{-1} e_{\mathbf{k}}\right)+\alpha \gamma \hbar^{-1} N_{0} c_{n}}{\left(i \omega_{n}\right)^{2}-\hbar^{-2} e_{\mathbf{k}}\left(e_{\mathbf{k}}+2 N_{0} c_{n}\right)} \\
{ }^{ \pm} \mathcal{G}_{\alpha \gamma} & =\frac{\delta_{\alpha \gamma}\left(\alpha i \omega_{n}+\hbar^{-1} e_{\mathbf{k}}\right)+\alpha \gamma \hbar^{-1} N_{0} c_{s}}{\left(i \omega_{n}\right)^{2}-\hbar^{-2} e_{\mathbf{k}}\left(e_{\mathbf{k}}+2 N_{0} c_{s}\right)}
\end{aligned}
$$

By the use of Eqs. (33) the correlation functions (40a), 42a and 44a can be cast to a form, after multiplying both the numerator and the denominator with ${ }^{n} \widetilde{\Delta}=\left(i \omega_{n}-\hbar^{-1} e_{\mathbf{k}}\right)\left(i \omega_{n}+\hbar^{-1} e_{\mathbf{k}}\right)$, where the denominators are common with the corresponding Green's functions in agreement with Eq. (86))

$$
\begin{aligned}
& { }^{0} \underline{\underline{D}}=\frac{\hbar^{-1} 2 N_{0} e_{\mathbf{k}}}{\left(i \omega_{n}\right)^{2}-\hbar^{-2} e_{\mathbf{k}}\left(e_{\mathbf{k}}+2 N_{0} c_{n}\right)}\left[\begin{array}{lll}
0 & 0 & 0 \\
0 & 1 & 0 \\
0 & 0 & 0
\end{array}\right] \\
& { }^{ \pm} \underline{\underline{D}}=\frac{1}{\left(i \omega_{n}\right)^{2}-\hbar^{-2} e_{\mathbf{k}}\left(e_{\mathbf{k}}+2 N_{0} c_{s}\right)}\left[\begin{array}{cc}
\mp N_{0}\left[i \omega_{n} \mp \hbar^{-1}\left(e_{\mathbf{k}}+N_{0} c_{s}\right)\right] & -c_{s} \hbar^{-1} N_{0}^{2} \\
-c_{s} \hbar^{-1} N_{0}^{2} & \pm N_{0}\left[i \omega_{n} \pm \hbar^{-1}\left(e_{\mathbf{k}}+N_{0} c_{s}\right)\right]
\end{array}\right] .
\end{aligned}
$$

With the use of Eqs. (70) the particle number and spin density correlation functions can also be calculated. The result is that $D_{n n}=2 \hbar^{-1} N_{0} e_{\mathbf{k}} /\left[\left(i \omega_{n}\right)^{2}-\hbar^{-2} e_{\mathbf{k}}\left(e_{\mathbf{k}}+2 N_{0} c_{n}\right)\right]$ while $D_{z z}=D_{n z}=0$. For spin waves $D_{++}=D_{--}=$ $4 \hbar^{-1} N_{0} e_{\mathbf{k}} /\left[\left(i \omega_{n}\right)^{2}-\hbar^{-2} e_{\mathbf{k}}\left(e_{\mathbf{k}}+2 N_{0} c_{s}\right)\right]$. From Eq. (89) and Eq. 68a) $D_{ \pm \pm}^{Q}=0$ as mentioned before.

\section{B. Ferromagnetic case}

In the ferromagnetic case $c_{s}<0$. One can choose the condensate spinor as $\zeta_{r}=\delta_{r,+}$. The calculation is analogous to that made in the previous subsection. The chemical potential (from Eq. (34)) is $\mu=N_{0}\left(c_{n}+c_{s}\right) \equiv N_{0} g$. The $\widetilde{\Sigma}_{11}^{r s}$ proper self-energy has 3 different components in its diagonal (other self-energy components are zero) which results in the following proper Green's functions:

$$
\begin{aligned}
\widetilde{\mathcal{G}}_{\alpha \gamma}^{++}\left(\mathbf{k}, i \omega_{n}\right) & =\frac{\delta_{\alpha \gamma}}{\alpha i \omega_{n}-\hbar^{-1} e_{\mathbf{k}}} \\
\widetilde{\mathcal{G}}_{\alpha \gamma}^{00}\left(\mathbf{k}, i \omega_{n}\right) & =\frac{\delta_{\alpha \gamma}}{\alpha i \omega_{n}-\hbar^{-1}\left(e_{\mathbf{k}}-N_{0} c_{s}\right)} \\
\widetilde{\mathcal{G}}_{\alpha \gamma}^{--}\left(\mathbf{k}, i \omega_{n}\right) & =\frac{\delta_{\alpha \gamma}}{\alpha i \omega_{n}-\hbar^{-1}\left(e_{\mathbf{k}}-2 N_{0} c_{s}\right)} .
\end{aligned}
$$

There are two things to note. The first one is that the $\mu_{0}$ parameter has cancelled out here as well, the second one is that the remaining self-energies (in the last two proper Green's functions) are negative since $c_{s}<0$ in the 
ferromagnetic case. The anomalous vertex vectors are:

$$
{ }_{f}^{0} \widetilde{\Lambda}_{\alpha}=\left(\begin{array}{c}
\sqrt{N_{0}} \\
0 \\
0
\end{array}\right), \quad+\underline{\tilde{\Lambda}}_{1}=\left(\begin{array}{c}
\sqrt{N_{0}} \\
0
\end{array}\right), \quad{ }_{f}^{+\widetilde{\Lambda}_{-1}}=\underline{0}
$$

The singular polarization matrices are:

$$
{ }^{0} \underline{\underline{\Pi}}^{(s)}=\left[\begin{array}{ccc}
\Pi_{++}^{(s)++} & 0 & 0 \\
0 & 0 & 0 \\
0 & 0 & 0
\end{array}\right], \quad{ }^{+} \underline{\underline{\Pi}}^{(s)}=\left[\begin{array}{cc}
\Pi_{+0}^{(s) 0+} & 0 \\
0 & 0
\end{array}\right], \quad{ }^{-} \underline{\underline{\Pi}}^{(s)}=\left[\begin{array}{cc}
\Pi_{0+}^{(s)+0} & 0 \\
0 & 0
\end{array}\right]
$$

with

$$
\begin{aligned}
& \Pi_{++}^{(s)++}=\hbar^{-1} N_{0}\left(\widetilde{\mathcal{G}}_{11}^{++}+\widetilde{\mathcal{G}}_{-1,-1}^{++}\right), \\
& \Pi_{+0}^{(s) 0+}=\hbar^{-1} N_{0} \widetilde{\mathcal{G}}_{1,1}^{00}, \\
& \Pi_{0+}^{(s)+0}=\hbar^{-1} N_{0} \widetilde{\mathcal{G}}_{-1,-1}^{00} .
\end{aligned}
$$

The singular polarization for the \pm 2 spin transfer reads as:

$$
\Pi_{ \pm \mp}^{(s) \mp \pm}=\hbar^{-1} N_{0} \widetilde{\mathcal{G}}_{ \pm 1, \pm 1}^{--}
$$

The improper self-energies are:

$$
{ }^{0} \underline{\underline{M}}=\hbar^{-1}\left[\begin{array}{ll}
N_{0} g & N_{0} g \\
N_{0} g & N_{0} g
\end{array}\right], \quad{ }^{+} M=M_{11}^{00}=\hbar^{-1} N_{0} c_{s}, \quad{ }^{Q} M=M_{11}^{--}=0,
$$

where $g=c_{n}+c_{s}$. For the ferromagnetic case the resulting Green's functions are (for the later ones see Eqs. (38)):

$$
\begin{aligned}
{ }^{0} \mathcal{G}_{\alpha \gamma} & =\mathcal{G}_{\alpha \gamma}^{++}=\frac{\delta_{\alpha \gamma}\left(\alpha i \omega_{n}+\hbar^{-1} e_{\mathbf{k}}\right)+\alpha \gamma \hbar^{-1} N_{0} g}{\left(i \omega_{n}\right)^{2}-\hbar^{-2} e_{\mathbf{k}}\left(e_{\mathbf{k}}+2 N_{0} g\right)} \\
{ }^{+} \mathcal{G}_{11} & =\mathcal{G}_{11}^{00}=\frac{1}{i \omega_{n}-\hbar^{-1} e_{\mathbf{k}}} \\
{ }^{Q} \mathcal{G}_{11} & =\mathcal{G}_{11}^{--}=\frac{1}{i \omega_{n}-\hbar^{-1}\left(e_{\mathbf{k}}-2 N_{0} c_{s}\right)}
\end{aligned}
$$

For spin transfers $n=0,+,-$ the correlation functions (40a), (42a) and (44a) can be evaluated from Eqs. (83) and with the use of the polarization matrices (100). Both the numerators and denominators are multiplied with the corresponding ${ }^{n} \widetilde{\Delta}$, leading to

$$
\begin{aligned}
{ }^{0} \underline{\underline{D}} & =\frac{2 \hbar^{-1} N_{0} e_{\mathbf{k}}}{\left(i \omega_{n}\right)^{2}-\hbar^{-2} e_{\mathbf{k}}\left(e_{\mathbf{k}}+2 N_{0} g\right)}\left[\begin{array}{lll}
1 & 0 & 0 \\
0 & 0 & 0 \\
0 & 0 & 0
\end{array}\right] \\
{ }^{+} \underline{\underline{D}} & =\frac{N_{0}}{i \omega_{n}-\hbar^{-1} e_{\mathbf{k}}}\left[\begin{array}{ll}
1 & 0 \\
0 & 0
\end{array}\right] \\
{ }^{-} \underline{\underline{D}} & =\frac{N_{0}}{-i \omega_{n}-\hbar^{-1} e_{\mathbf{k}}}\left[\begin{array}{ll}
1 & 0 \\
0 & 0
\end{array}\right]
\end{aligned}
$$

The correlation functions with $n= \pm 2$ obtained directly from Eqs. (46) and (47) with polarization functions (100e) read as

$$
D_{ \pm \mp}^{\mp \pm}=\frac{N_{0}}{ \pm i \omega_{n}-\hbar^{-1}\left(e_{\mathbf{k}}-2 N_{0} c_{s}\right)}
$$


The particle number and spin density correlation functions from Eqs. (70) are:

$$
\begin{aligned}
D_{n n} & =D_{z z}=D_{n z}=\frac{2 \hbar^{-1} N_{0} e_{\mathbf{k}}}{\left(i \omega_{n}\right)^{2}-\hbar^{-2} e_{\mathbf{k}}\left(e_{\mathbf{k}}+2 N_{0} g\right)}, \\
D_{++} & =\frac{2 N_{0}}{i \omega_{n}-\hbar^{-1} e_{\mathbf{k}}}, \\
D_{--} & =\frac{2 N_{0}}{-i \omega_{n}-\hbar^{-1} e_{\mathbf{k}}}, \\
D_{ \pm \pm}^{Q} & =\frac{4 N_{0}}{ \pm i \omega_{n}-\hbar^{-1}\left(e_{\mathbf{k}}-2 N_{0} c_{s}\right)} .
\end{aligned}
$$

\section{Collective excitations in the Bogoliubov approximation}

The spectra of collective excitations can be expressed using Eq. (88) (for the 0 and \pm 1 spin transfer modes) or equivalently by the zeroes of the denominators of the appropriate retarded correlation functions. For the polar case this results in

$$
\begin{gathered}
{ }^{0} \omega=\hbar^{-1} \sqrt{e_{\mathbf{k}}\left(e_{\mathbf{k}}+2 N_{0} c_{n}\right)} \underset{k \rightarrow 0}{\rightarrow} \sqrt{\frac{N_{0} c_{n}}{M}} k, \\
{ }^{ \pm} \omega=\hbar^{-1} \sqrt{e_{\mathbf{k}}\left(e_{\mathbf{k}}+2 N_{0} c_{s}\right)} \underset{k \rightarrow 0}{\rightarrow} \sqrt{\frac{N_{0} c_{s}}{M}} k .
\end{gathered}
$$

There are no other excitation modes for the polar case in this approximation. These modes have linear spectrum and are Goldstone modes. The ${ }^{0} \omega$ mode belongs to the ${ }^{0} \mathcal{G}_{\alpha \gamma}$ Green's functions and the $D_{n n}\left({ }^{0} \underline{\underline{D}}\right)$ correlation functions, while the ${ }^{ \pm} \omega$ mode belongs to the ${ }^{ \pm} \mathcal{G}_{\alpha \gamma}$ Green's functions and the $D_{ \pm \pm}\left({ }^{ \pm} \underline{\underline{D}}\right)$ correlation functions.

For the ferromagnetic case the excitational energies are at:

$$
\begin{aligned}
{ }^{0} \omega & =\hbar^{-1} \sqrt{e_{\mathbf{k}}\left(e_{\mathbf{k}}+2 N_{0} g\right)} \underset{k \rightarrow 0}{\rightarrow} \sqrt{\frac{N_{0} g}{M}} k, \\
{ }^{ \pm} \omega & = \pm \hbar^{-1} e_{\mathbf{k}}, \\
{ }^{ \pm} \omega & = \pm \hbar^{-1}\left(e_{\mathbf{k}}-2 N_{0} c_{s}\right) .
\end{aligned}
$$

The first $\left({ }^{0} \omega\right)$ mode, which is responsible to density (and spin density) fluctuations, belongs to the ${ }^{0} \mathcal{G}_{\alpha \gamma}$ Green's functions and the connected $D_{n n}, D_{z z}$ and $D_{n z}\left({ }^{0} \underline{\underline{D}}\right)$ correlation functions. This is a linear Goldstone mode. The next $\left({ }^{ \pm} \omega\right)$ modes are responsible for ordinary spin waves and belong to the ${ }^{ \pm} \mathcal{G}_{11}=\mathcal{G}_{ \pm \pm}^{00}$ Green's functions and the $D_{ \pm \pm}\left({ }^{ \pm} \underline{\underline{D}}\right)$ correlation functions. These are also Goldstone modes but with a quadratic dispersion. The last $\left({ }_{Q}^{ \pm} \omega\right)$ modes describe quadrupolar spin waves. They belong to the ${ }_{Q}^{ \pm} \mathcal{G}_{11}=\mathcal{G}_{ \pm \pm}^{--}$Green's functions and the $D_{ \pm \pm}^{Q}\left(D_{ \pm \mp}^{\mp \pm}\right)$ correlation functions. These are non Goldstone modes and they start with a gap. The frequencies found in the Bogoliubov approximation agree with those of Refs. 14, 15] obtained in a different manner.

\section{RANDOM PHASE APPROXIMATION}

The simplest way to take into account the appearance of the noncondensed atoms, leading to a damping mechanism, is to add a Hartree term to the Bogoliubov proper self-energies (compare Fig. 14 with Fig. 16) and to choose the regular polarization graphs as bubbles. Some results of this approximation were published earlier in [41].

Therefore the proper self-energies of the random phase approximation can be graphically represented as seen in Fig. 16 and their contributions are

$$
\begin{aligned}
& \widetilde{\Sigma}_{\alpha \gamma}^{r s}=\hbar^{-1}\left[\left(\mu_{0}-\mu\right) \delta_{r s} \delta_{\alpha \gamma}+N_{0} \zeta_{r^{\prime}}^{\dagger} V_{r s}^{r^{\prime} s^{\prime}} \zeta_{s^{\prime}}+H^{s^{\prime} r^{\prime}} V_{r s}^{r^{\prime} s^{\prime}}\right] \\
& \widetilde{\Sigma}_{01}^{s}=\hbar^{-1} \sqrt{N_{0}}\left[(-\mu) \zeta_{r}^{\dagger} \delta_{r s}+N_{0} \zeta_{r^{\prime}} \zeta_{r}^{\dagger} V_{r s}^{r^{\prime} s^{\prime}}+H^{s^{\prime} r^{\prime}} V_{r s}^{r^{\prime} s^{\prime}}\right]
\end{aligned}
$$


Here the $H^{s r}$ notation is introduced for the contribution of the Hartree term:

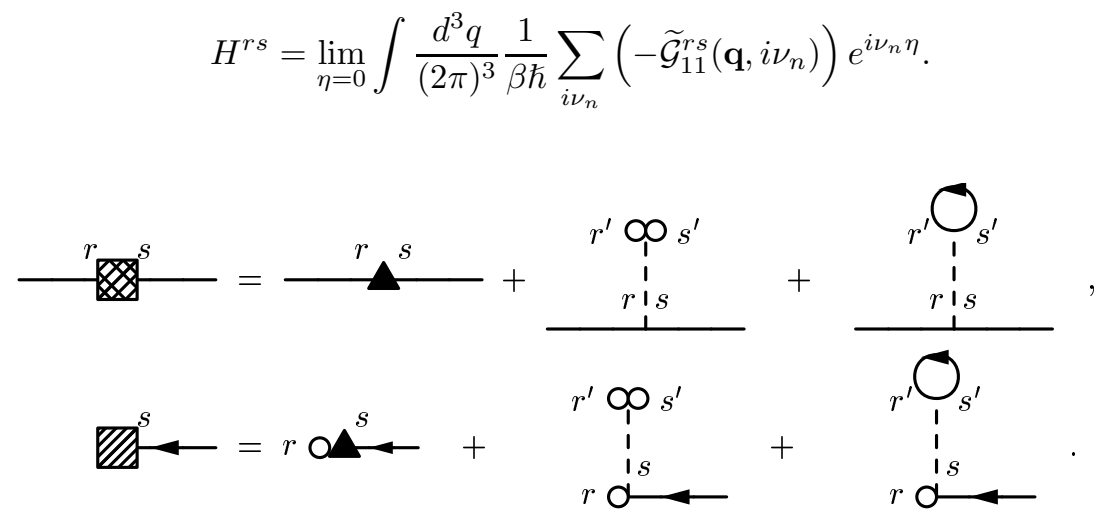

FIG. 16. The graphical representation of the proper self-energies and the tadpole graphs in the RPA

The proper self-energies are independent of the wave-numbers and frequencies and diagonal both in the Roman and Greek indices. The proper Green's functions are diagonal in the Roman and Greek indices as well. The approximation is done in a self-consistent way, namely the internal propagators used are proper Green's functions in the Hartree approximation.

The regular polarization will be a bubble (with proper Green's functions) as seen in Fig. 17. Its contribution is:

$$
-\hbar \Pi_{r^{\prime} s^{\prime}}^{(r) s r}\left(\mathbf{k}, i \omega_{n}\right)=\int \frac{d^{3} q}{(2 \pi)^{3}} \frac{1}{\beta \hbar} \sum_{i \nu_{n}} \widetilde{\mathcal{G}}_{11}^{r, r^{\prime}}\left(\mathbf{q}, i \nu_{n}\right) \widetilde{\mathcal{G}}_{11}^{s^{\prime} s}\left(\mathbf{k}+\mathbf{q}, i \omega_{n}+i \nu_{n}\right) .
$$

Since our approximate proper Green's functions are diagonal in their spin indices $\left(r=r^{\prime}\right.$ and $\left.s=s^{\prime}\right)$ the regular polarization matrices will be diagonal as well, with

$$
\begin{aligned}
& { }^{0} \underline{\underline{\Pi}}^{(r)}=\left[\begin{array}{ccc}
\Pi_{++}^{(r)++} & 0 & 0 \\
0 & \Pi_{00}^{(r) 00} & 0 \\
0 & 0 & \Pi_{--}^{(r)--}
\end{array}\right], \quad{ }^{+} \underline{\underline{\Pi}}^{(r)}=\left[\begin{array}{cc}
\Pi_{+0}^{(r) 0+} & 0 \\
0 & \Pi_{0-}^{(r)-0}
\end{array}\right] . \\
& \underbrace{r}_{s}=\overbrace{s}^{r_{s^{\prime}}^{\prime}}
\end{aligned}
$$

FIG. 17. The Feynamn graph of the regular polarization function in the random phase (Hartree) approximation

The anomalous vertex remains the same as it was in the Bogoliubov approximation, see Fig 15 and Eq. (91). With the building blocks determined we can now turn our attention to the construction of the correlation functions.

\section{A. Polar case}

Using the interaction (7) with $c_{s}>0$ and $\zeta_{r}=\delta_{r, 0}$ the self-energies (107) in the polar case

$$
\begin{aligned}
\widetilde{\Sigma}_{11}^{++} & =\hbar^{-1}\left[\mu_{0}+\left(H^{++}-H^{--}\right) c_{s}\right], \\
\widetilde{\Sigma}_{11}^{00} & =\hbar^{-1} \mu_{0}, \\
\widetilde{\Sigma}_{11}^{--} & =\hbar^{-1}\left[\mu_{0}-\left(H^{++}-H^{--}\right) c_{s}\right] .
\end{aligned}
$$

Since in the polar case the system has zero magnetisation the Hartree terms should satisfy $H^{++}=H^{--}$and as a consequence the proper Green's functions are the same as they were in the Bogoliubov approximation Eq. (92). The situation is similar to that in the scalar case, the chemical potential cancels the Hartree terms [31]. 
The anomalous vertex vectors are given by Eq. (93), and so the singular polarization matrices will also be the same as for the Bogoliubov approximation Eq. (94). The regular polarization matrices are as follows:

$$
{ }^{0} \underline{\underline{\Pi}}^{(r)}=\left[\begin{array}{ccc}
\Pi_{0} & 0 & 0 \\
0 & \Pi_{0} & 0 \\
0 & 0 & \Pi_{0}
\end{array}\right], \quad{ }^{+} \underline{\underline{\Pi}}^{(r)}=\left[\begin{array}{cc}
\Pi_{0} & 0 \\
0 & \Pi_{0}
\end{array}\right]
$$

in accordance with the spin reflection symmetry discussed in Sec. III . Here the notation $\Pi_{0} \equiv \Pi_{++}^{(r)++}=\Pi_{00}^{(r) 00}=$ $\Pi_{--}^{(r)--}$ is introduced. The interaction propagators are then obtained from Eqs. (66a) and (66b),

$$
{ }^{0} \underline{\underline{W}}=\left[\begin{array}{ccc}
\mathcal{C}_{n}^{(r)}+\mathcal{C}_{s}^{(r)} & \mathcal{C}_{n}^{(r)} & \mathcal{C}_{n}^{(r)}-\mathcal{C}_{s}^{(r)} \\
\mathcal{C}_{n}^{(r)} & \mathcal{C}_{n}^{(r)} & \mathcal{C}_{n}^{(r)} \\
\mathcal{C}_{n}^{(r)}-\mathcal{C}_{s}^{(r)} & \mathcal{C}_{n}^{(r)} & \mathcal{C}_{n}^{(r)}-\mathcal{C}_{s}^{(r)}
\end{array}\right], \quad \quad \underline{\underline{W}}=\left[\begin{array}{cc}
\mathcal{C}_{s}^{(r)} & \mathcal{C}_{s}^{(r)} \\
\mathcal{C}_{s}^{(r)} & \mathcal{C}_{s}^{(r)}
\end{array}\right]
$$

with

$$
\mathcal{C}_{n}^{(r)}\left(\mathbf{k}, i \omega_{n}\right)=\frac{c_{n}}{1-3 c_{n} \Pi_{0}\left(\mathbf{k}, i \omega_{n}\right)}, \quad \mathcal{C}_{s}^{(r)}\left(\mathbf{k}, i \omega_{n}\right)=\frac{c_{s}}{1-2 c_{s} \Pi_{0}\left(\mathbf{k}, i \omega_{n}\right)}
$$

With Eq. (67) the improper self-energies become

$$
{ }^{0} \underline{\underline{M}}=\hbar^{-1}\left[\begin{array}{cc}
N_{0} \mathcal{C}_{n}^{(r)} & N_{0} \mathcal{C}_{n}^{(r)} \\
N_{0} \mathcal{C}_{n}^{(r)} & N_{0} \mathcal{C}_{n}^{(r)}
\end{array}\right], \quad{ }^{ \pm} \underline{\underline{M}}=\hbar^{-1}\left[\begin{array}{ccc}
N_{0} \mathcal{C}_{s}^{(r)} & N_{0} \mathcal{C}_{s}^{(r)} \\
N_{0} \mathcal{C}_{s}^{(r)} & N_{0} \mathcal{C}_{s}^{(r)}
\end{array}\right]
$$

These can be used in Eqs. (82) to arrive at the Green's functions in RPA. After multiplying both the numerator and denominator with $\operatorname{det}^{n} \underline{\underline{\underline{\varepsilon}}}(r)$ we obtain

$$
\begin{aligned}
{ }^{0} \mathcal{G}_{\alpha \gamma} & =\frac{\delta_{\alpha \gamma}\left(\alpha i \omega_{n}+\hbar^{-1} e_{\mathbf{k}}\right)\left(1-3 c_{n} \Pi_{0}\right)+\alpha \gamma \hbar^{-1} N_{0} c_{n}}{\left[\left(i \omega_{n}\right)^{2}-\hbar^{-2} e_{\mathbf{k}}^{2}\right]\left(1-3 c_{n} \Pi_{0}\right)-2 \hbar^{-2} e_{\mathbf{k}} N_{0} c_{n}}, \\
{ }^{ \pm} \mathcal{G}_{\alpha \gamma} & =\frac{\delta_{\alpha \gamma}\left(\alpha i \omega_{n}+\hbar^{-1} e_{\mathbf{k}}\right)\left(1-2 c_{s} \Pi_{0}\right)+\alpha \gamma \hbar^{-1} N_{0} c_{s}}{\left[\left(i \omega_{n}\right)^{2}-\hbar^{-2} e_{\mathbf{k}}^{2}\right]\left(1-2 c_{s} \Pi_{0}\right)-2 \hbar^{-2} e_{\mathbf{k}} N_{0} c_{s}} .
\end{aligned}
$$

Calculating the regular dielectric functions from Eqs. (65) and using Eqs. 83 the correlation function matrices could be explicitly given. But in the polar case one can use Eqs. (74) to calculate directly the relevant correlation functions in the zero spin transfer, with $\Pi_{n n}=3 c_{n} \Pi_{0}+c_{n} \Pi_{S}$ and $\Pi_{z z}=2 c_{s} \Pi_{0}$ leading to

$$
\begin{aligned}
D_{n n} & =\hbar \frac{3 \Pi_{0}\left[\left(i \omega_{n}\right)^{2}-\hbar^{-2} e_{\mathbf{k}}^{2}\right]+2 \hbar^{-2} N_{0} e_{\mathbf{k}}}{\left[\left(i \omega_{n}\right)^{2}-\hbar^{-2} e_{\mathbf{k}}^{2}\right]\left(1-3 c_{n} \Pi_{0}\right)-2 \hbar^{-2} e_{\mathbf{k}} N_{0} c_{n}}, \\
D_{z z} & =\hbar \frac{2 \Pi_{0}}{1-2 c_{s} \Pi_{0}} \\
D_{n z} & =0 .
\end{aligned}
$$

With Eq. (71) and with $\Pi_{ \pm \pm}=4 \Pi_{0}+2 \Pi_{S}$ the correlation function of the spin waves will become

$$
D_{ \pm \pm}=\hbar \frac{4 \Pi_{0}\left[\left(i \omega_{n}\right)^{2}-\hbar^{-2} e_{\mathbf{k}}^{2}\right]+2 \hbar^{-2} N_{0} e_{\mathbf{k}}}{\left[\left(i \omega_{n}\right)^{2}-\hbar^{-2} e_{\mathbf{k}}^{2}\right]\left(1-2 c_{s} \Pi_{0}\right)-2 \hbar^{-2} e_{\mathbf{k}} N_{0} c_{s}} .
$$

The correlation functions of the quadrupolar spin waves come from Eq. (89) with a result of

$$
D_{ \pm \pm}^{Q}=\hbar 4 \Pi_{0}
$$

One can easily verify that the denominators of the appropriate correlation functions match as was shown at the general formalism. 


\section{B. Ferromagnetic case}

In this phase $c_{s}<0$ and $\zeta_{r}=\delta_{r,+}$ and with the interaction (7) the self-energies (107) become

$$
\begin{aligned}
\widetilde{\Sigma}_{11}^{++} & =\hbar^{-1} \mu_{0}, \\
\widetilde{\Sigma}_{11}^{00} & =\hbar^{-1}\left(\mu_{0}-\mathcal{M} c_{s}\right), \\
\widetilde{\Sigma}_{11}^{--} & =\hbar^{-1}\left(\mu_{0}-2 \mathcal{M} c_{s}\right),
\end{aligned}
$$

where the newly introduced quantity

$$
\mathcal{M}=N_{0}+H^{++}-H^{--}
$$

is the total magnetisation of the system. It describes the number of particles responsible for the magnetic mean-field. Note that since the Hartree terms are momentum independent the proper self-energies will also be. Therefore the proper Green's functions for the ferromagnetic case read as

$$
\begin{aligned}
\widetilde{\mathcal{G}}_{\alpha \gamma}^{++}\left(\mathbf{k}, i \omega_{n}\right) & =\frac{\delta_{\alpha \gamma}}{\alpha i \omega_{n}-\hbar^{-1} e_{\mathbf{k}}}, \\
\widetilde{\mathcal{G}}_{\alpha \gamma}^{00}\left(\mathbf{k}, i \omega_{n}\right) & =\frac{\delta_{\alpha \gamma}}{\alpha i \omega_{n}-\hbar^{-1}\left(e_{\mathbf{k}}-\mathcal{M} c_{s}\right)}, \\
\widetilde{\mathcal{G}}_{\alpha \gamma}^{--}\left(\mathbf{k}, i \omega_{n}\right) & =\frac{\delta_{\alpha \gamma}}{\alpha i \omega_{n}-\hbar^{-1}\left(e_{\mathbf{k}}-2 \mathcal{M} c_{s}\right)} .
\end{aligned}
$$

These proper Green's functions are similar to their Bogoliubov counterparts only the momentum independent mean field has changed with the appearance of the noncondensed particles with spin projection + and - . The chemical potential here do not fully cancels the proper self-energies leaving behind the energy shift due to the magnetic mean field.

The anomalous vertex vectors are given by Eqs. (99). One can express the singular polarization matrices from Eqs. (68) for $n=0,+,-$, and they are of the same form as their Bogoliubov counterparts (100a) with elements (100b), (100d) and (100d) where the proper Green's functions (118) are to be used. The singular polarization for the \pm 2 spin transfer can also be brought to the form (100) with (1189) as the proper Green's function,

$$
\Pi_{ \pm \mp}^{(s) \mp \pm}=\hbar^{-1} N_{0} \widetilde{\mathcal{G}}_{ \pm 1, \pm 1}^{--} .
$$

Using Eqs. (65) and Eq. (110) the effective potential $W$ can be constructed. Inserting it to Eqs. (67) the improper self-energies will read as

$$
{ }^{0} \underline{\underline{M}}=\frac{\hbar^{-1} N_{0} \rho}{\operatorname{det}^{0} \underline{\underline{\varepsilon}}^{(r)}}\left[\begin{array}{ll}
1 & 1 \\
1 & 1
\end{array}\right], \quad{ }^{+} \underline{\underline{M}}=\frac{\hbar^{-1} N_{0} c_{s}}{\operatorname{det}^{+} \underline{\underline{\varepsilon}}}\left[\begin{array}{ll}
1 & 0 \\
0 & 0
\end{array}\right], \quad{ }^{(r)} M=M_{11}^{--}=0,
$$

where the following notations are introduced to simplify the equations:

$$
\begin{aligned}
\rho & =c_{n}+c_{s}-c_{n} c_{s}\left(4 \Pi_{--}^{(r)--}+\Pi_{00}^{(r) 00}\right), \\
\operatorname{det}^{0} \underline{\underline{\varepsilon}}^{(r)} & =\left(1-c_{n} \Pi_{n n}^{(r)}\right)\left(1-c_{s} \Pi_{z z}^{(r)}\right)-c_{n} c_{s} \Pi_{n z}^{(r)}{ }^{2} \\
\operatorname{det}^{+} \underline{\underline{\varepsilon}}^{(r)} & =1-\frac{c_{s}}{2} \Pi_{++}^{(r)},
\end{aligned}
$$

with

$$
\begin{aligned}
\Pi_{n n}^{(r)} & =\Pi_{++}^{(r)++}+\Pi_{00}^{(r) 00}+\Pi_{--}^{(r)--} \\
\Pi_{z z}^{(r)} & =\Pi_{++}^{(r)++}+\Pi_{--}^{(r)--} \\
\Pi_{n z}^{(r)} & =\Pi_{++}^{(r)++}-\Pi_{--}^{(r)--} \\
\Pi_{++}^{(r)} & =2\left(\Pi_{+0}^{(r) 0+}+\Pi_{0+}^{(r)+0}\right) .
\end{aligned}
$$


From Eqs. (82) and from Eq. (38b) the Green's functions are

$$
\begin{aligned}
{ }^{0} \mathcal{G}_{\alpha \gamma} & =\mathcal{G}_{\alpha \gamma}^{++}=\frac{\delta_{\alpha \gamma}\left(\alpha i \omega_{n}+\hbar^{-1} e_{\mathbf{k}}\right) \operatorname{det}^{0} \underline{\underline{\varepsilon}}^{(r)}+\alpha \gamma \hbar^{-1} N_{0} \rho}{\left[\left(i \omega_{n}\right)^{2}-\hbar^{-2} e_{\mathbf{k}}^{2}\right] \operatorname{det}{ }^{0} \underline{\underline{\varepsilon}}^{(r)}-2 \hbar^{-2} e_{\mathbf{k}} N_{0} \rho}, \\
{ }^{+} \mathcal{G}_{11} & =\mathcal{G}_{11}^{00}=\frac{\operatorname{det}^{+} \underline{\underline{\varepsilon}}^{(r)}}{\left[i \omega_{n}-\hbar^{-1}\left(e_{\mathbf{k}}-\mathcal{M} c_{s}\right)\right] \operatorname{det}{ }^{+} \underline{\underline{\varepsilon}}^{(r)}-\hbar^{-1} N_{0} c_{s}}, \\
{ }^{Q} \mathcal{G}_{11} & =\mathcal{G}_{11}^{--}=\frac{1}{i \omega_{n}-\hbar^{-1}\left(e_{\mathbf{k}}-2 \mathcal{M} c_{s}\right)} .
\end{aligned}
$$

Equations (83a) and (89) give the correlation function matrices which combined with Eqs. (70) result in

$$
\begin{aligned}
D_{n n} & =\hbar \frac{\left(\Pi_{++}^{(r)++}+\Pi_{++}^{(s)++}\right)\left[1-c_{s}\left(\Pi_{00}^{(r) 00}+4 \Pi_{--}^{(r)--}\right)\right]+\Pi_{00}^{(r) 00}+\Pi_{--}^{(r)--}\left(1-c_{s} \Pi_{00}^{(r) 00}\right)}{\operatorname{det}^{0} \underline{\underline{\varepsilon}}}, \\
D_{z z} & =\hbar \frac{\left(\Pi_{++}^{(r)++}+\Pi_{++}^{(s)++}\right)\left[1-c_{n}\left(\Pi_{00}^{(r) 00}+4 \Pi_{--}^{(r)-}\right)\right]+\Pi_{--}^{(r)--}\left(1-c_{n} \Pi_{00}^{(r) 00}\right)}{\operatorname{det}^{0} \underline{\underline{\varepsilon}}}, \\
D_{n z} & =\hbar \frac{\Pi_{++}^{(r)++}+\Pi_{++}^{(s)++}-\Pi_{--}^{(r)--}}{\operatorname{det}^{0} \underline{\underline{\varepsilon}}}, \\
D_{++} & =2 \hbar \frac{\Pi_{+0}^{(r) 0+}+\Pi_{+0}^{(s) 0+}+\Pi_{0+}^{(r)+0}}{\operatorname{det}^{+} \underline{\underline{\varepsilon}}}, \\
D_{++}^{Q} & =4 \hbar\left(\Pi_{+-}^{(r)-+}+\Pi_{+-}^{(s)-+}\right) .
\end{aligned}
$$

Here

$$
\begin{aligned}
\operatorname{det}^{0} \underline{\underline{\varepsilon}} & =\left(1-c_{n} \Pi_{n n}\right)\left(1-c_{s} \Pi_{z z}\right)-c_{n} c_{s} \Pi_{n z}^{2}, \\
\operatorname{det}^{+} \underline{\underline{\varepsilon}} & =1-\frac{c_{s}}{2} \Pi_{++},
\end{aligned}
$$

where $\Pi_{n n}=\Pi_{n n}^{(r)}+\Pi_{++}^{(s)++}, \Pi_{z z}=\Pi_{z z}^{(r)}+\Pi_{++}^{(s)++}, \Pi_{n z}=\Pi_{n z}^{(r)}+\Pi_{++}^{(s)++}$ and $\Pi_{++}=\Pi_{++}^{(r)}+\Pi_{+0}^{(s) 0+}$. Equations (122a), (122b), (122c) and (122d can be cast to a form having common denominators with the corresponding Green's functions by multiplying both their numerators and denominators with the apropriate ${ }^{n} \widetilde{\Delta}$. Only writig down the resulting denominators:

$$
\begin{aligned}
{ }^{0} \widetilde{\Delta} \operatorname{det}^{0} \underline{\underline{\varepsilon}} & =\left[\left(i \omega_{n}\right)^{2}-\hbar^{-2} e_{\mathbf{k}}^{2}\right] \operatorname{det}^{0} \underline{\underline{\varepsilon}}^{(r)}-2 \hbar^{-1} e_{\mathbf{k}} N_{0} \rho \\
& +\widetilde{\Delta} \operatorname{det}^{+} \underline{\underline{\varepsilon}}=\left[\left(i \omega_{n}\right)-\hbar^{-1}\left(e_{\mathbf{k}}-\mathcal{M} c_{s}\right)\right] \operatorname{det}^{+} \underline{\underline{\varepsilon}}^{(r)}-\hbar^{-1} N_{0} c_{s}
\end{aligned}
$$

For the rest of the paper we will deal with the retarded correlation functions which can be obtained in the usual way by analytically continuing in frequencies (see e.g. Ref. [40]). First however we will discuss shortly the static properties.

\section{Static properties of the spin-1 Bose gas in the random phase approximation}

We will study the equation of state and connect it to the density autocorrelation function through the compressibility sum rule. The equation of state is investigated by choosing $\mu, N$ as conjugated variables. The number of particles $N$ is given by

$$
N=N_{0}+H^{s s}
$$

where $H^{s r}$ is defined by Eq. (108). In the ordered phase Eqs. (34), (117d) and (124) provide a second relationship

$$
\mu=c_{n} N+c_{s} \mathcal{M} .
$$


It is convenient to introduce the following quantities: the thermal wavelength $\lambda=\hbar(\beta / 2 M)^{1 / 2}$, the critical particle density of the ideal gas $N_{c}=3 \Gamma\left(\frac{3}{2}\right) \zeta\left(\frac{3}{2}\right) /\left[(2 \pi)^{2} \lambda^{3}\right]$, dimensionless interaction strengths $\epsilon_{n, s}=N_{c} \beta\left|c_{n, s}\right|$, relative total magnetisation $m=\mathcal{M} / N_{c}$, relative particle number $x=N / N_{c}$ and dimensionless chemical potential $u=\beta \mu$. Here $\Gamma(s)$ is the Gamma function and $\zeta(s)$ is the Riemann-zeta function.

Both for the polar and ferromagnetic cases in the symmetric phase (where no condensate is present and the total magnetisation is zero) the equation of state reads as

$$
x=\frac{1}{\zeta\left(\frac{3}{2}\right)} F\left(\frac{3}{2}, x \epsilon_{n}-u\right),
$$

where

$$
F(s, \gamma)=\frac{1}{\Gamma(s)} \int_{0}^{\infty} \frac{t^{s-1}}{e^{t+\gamma}-1} d t
$$

is the Bose-Einstein integral.

In the condensed phase for the polar case the chemical potential is determined by Eq. (34) which leads to

$$
u=\epsilon_{n} x .
$$

Equations $(126)$ and $(128)$ can be used to give the isotherms $u(x)$ of the polar case. Equation $(126)$ is valid when $x<1$ and Eq. (128) is to be used if $x>1$. These isotherms show the character of a continuous phase transition.

In the condensed phase for the ferromagnetic case the chemical potential can be calculated also from Eq. (34)

$$
u=\epsilon_{n} x-\epsilon_{s} m(x)=\epsilon_{s}[\kappa x-m(x)],
$$

where $\kappa=\epsilon_{n} / \epsilon_{s}$ and $m(x)$ is the relative total magnetisation as a function of the relative total density given by the

$$
m=x-\frac{1}{3 \zeta\left(\frac{3}{2}\right)}\left[F\left(\frac{3}{2}, m \epsilon_{s}\right)+2 F\left(\frac{3}{2}, 2 m \epsilon_{s}\right)\right]
$$

equation. The isotherms are given by Eqs. (126), 129a and (129b). The solution of Eq. (129b) is unique if $x>1$ but at $x<1$ a second solution emerges also with nonzero magnetization and both solutions can be continued down to $x_{v}<1$, where they coincide and vanish. This means that the chemical potential has three solutions between $x=x_{v}$ and $x=1$. (One of them with zero magnetization at a given density.) In the applications $\epsilon_{s} \ll 1$, so the Bose-Einstein integral can be well approximated by $F\left(\frac{3}{2}, \alpha\right) \approx \zeta\left(\frac{3}{2}\right)-2 \sqrt{\pi \alpha}$ leading to

$$
x_{v}=1-\frac{(1+2 \sqrt{2})^{2} \pi \epsilon_{s}}{9 \zeta\left(\frac{3}{2}\right)^{2}} .
$$

At $x=1$ the two solutions are at

$$
m_{1,2}(x=1)=\left\{\begin{array}{c}
\frac{4(1+2 \sqrt{2})^{2} \pi \epsilon_{s}}{9 \zeta\left(\frac{3}{2}\right)^{2}}, \\
0 .
\end{array}\right.
$$

These isotherms show the character of a very weak first order phase transition. A similar equation of state with a first order transition was derived in [39] for scalar condensates but in a different model approximation. In that work the first order character of the phase transition was caused by the exchange interaction (Fock term) which is known to lead to such behaviour [42. The situation is interesting, since for scalar particles the RPA (Hartree approximation) gave a continuous phase transition as here for the polar case [31,30].

Since the above behaviour of the equation of state is not common a further consistency check is required. The compressibility sum rule is a candidate, since it relates the static correlation functions to the derivatives of the equation of state 43, 39], namely

$$
\left(\frac{\partial N}{\partial \mu}\right)_{T}=\hbar^{-1} D_{n n}(k \rightarrow 0, \omega=0) .
$$

The inverse of the left hand side of Eq. (132) can be easily calculated, since

$$
\left(\frac{\partial \mu}{\partial N}\right)_{T}=\frac{1}{\beta N_{c}} u^{\prime}(x)=\frac{1}{\beta N_{c}}\left[\kappa \epsilon_{s}-m^{\prime}(x) \epsilon_{s}\right] .
$$


The derivative $m^{\prime}(x)$ can be expressed from Eq. (129b) with the identity $\partial_{\alpha} F(s, \alpha)=-F(s-1, \alpha)$, leading to

$$
\left(\frac{\partial \mu}{\partial N}\right)_{T}=\frac{c_{n}+c_{s}-c_{n} c_{s}\left[\Pi_{00}^{(r) 00}(0,0)+4 \Pi_{--}^{(r)--}(0,0)\right]}{1-c_{s}\left[\Pi_{00}^{(r) 00}(0,0)+4 \Pi_{--}^{(r)--}(0,0)\right]}
$$

where we used that $\Pi_{00}^{(r) 00}(0,0)=-\beta N_{c} F\left(\frac{1}{2}, m \epsilon_{s}\right) /\left(3 \zeta\left(\frac{3}{2}\right)\right)$ and $\Pi_{--}^{(r)--}(0,0)=-\beta N_{c} F\left(\frac{1}{2}, 2 m \epsilon_{s}\right) /\left(3 \zeta\left(\frac{3}{2}\right)\right)$. It is straightforward to verify that the static limit of $\hbar / D_{n n}$ is just the right hand side of Eq. (134) in agreement with Eq. (132). The point $x_{v}$ where the two solutions $m_{1}$ and $m_{2}$ coincide is the point where the graph of $u(x)$ has a vertical tangent. The point where the graph of $u(x)$ has a horizontal tangent is at where the density autocorrelation function has its pole at $q=0$ and $\omega=0$. This happens at

$$
x_{h}=1-\frac{\kappa^{2}-2 \kappa}{(\kappa-1)^{2}} \frac{(1+2 \sqrt{2})^{2} \pi \epsilon_{s}}{9 \zeta\left(\frac{3}{2}\right)^{2}} .
$$

For $x>x_{h}$ (in the condensed phase) the static correlation functions have no real positive pole in the $q$ variable.

\section{Collective excitations in the random phase approximation}

The excitation spectra can be obtained in the usual way as the poles of the retarded Green's functions and correlation functions. The real parts of the poles correspond to the energies while the imaginary parts correspond to the damping (the inverse of their lifetimes) of the excitations.

Our further calculations can be done more appropriately in a dimensionless form. For this reason let us further introduce the following characteristic lengths: $\xi_{n, s}^{B}=\hbar\left(4 M N_{0}\left|c_{n, s}\right|\right)^{-1 / 2}$ the mean field correlation lengths associated with the interaction strength $c_{n}$ and $c_{s}$, and $\xi^{\prime}=M /\left(4 \pi \hbar^{2} N_{0} \beta\right)$ the length scale of the critical fluctuations. The calculation will be limited to the intermediate temperature region. This region is defined by the $\xi_{n, s}^{B} \gg \xi^{\prime}, \lambda$ conditions. In this temperature range the $k \lambda \ll 1$ condition can be fulfilled for the physically interesting wave numbers and the contribution of the bubble diagrams can be taken as perturbation for the frequencies considered.

A dimensionless frequency can be introduced with

$$
\Omega=\frac{\hbar \omega}{e_{\mathbf{k}}} .
$$

The singular polarization functions in the polar case take the following form:

$$
c_{n, s} \Pi_{S}(\mathbf{k}, \omega)=\left(\frac{\lambda}{\xi_{n, s}^{B}}\right)^{2} \frac{1}{(k \lambda)^{2}} \frac{1}{\Omega^{2}-1} .
$$

While in the ferromagnetic case they read as

$$
\begin{aligned}
\left|c_{n, s}\right| \Pi_{++}^{(s)++}(\mathbf{k}, \omega) & =\left(\frac{\lambda}{\xi_{n, s}^{B}}\right)^{2} \frac{1}{(k \lambda)^{2}} \frac{1}{\Omega^{2}-1} \\
\left|c_{s}\right| \Pi_{+0}^{(s) 0+}(\mathbf{k}, \omega) & =\frac{\gamma_{0}}{(k \lambda)^{2}(\Omega-1)-\widetilde{\gamma}} \\
\left|c_{s}\right| \Pi_{+-}^{(s)-+}(\mathbf{k}, \omega) & =\frac{\gamma_{0}}{(k \lambda)^{2}(\Omega-1)-2 \widetilde{\gamma}}
\end{aligned}
$$

with

$$
\begin{aligned}
\gamma_{0} & =\beta N_{0}\left|c_{s}\right| \\
\widetilde{\gamma} & =\beta \mathcal{M}\left|c_{s}\right|
\end{aligned}
$$

For $k \lambda \ll 1$ and $|\Omega k \lambda| \ll 1$ the regular polarization function (the bubble) in the polar case can be approximated as

$$
c_{n, s} \Pi_{0}(\mathbf{k}, \omega) \approx \frac{\left(\lambda \xi^{\prime}\right)}{\left(\xi_{n, s}^{B}\right)^{2}} \frac{1}{k \lambda} \frac{i}{2} \ln \frac{\Omega-1}{\Omega+1},
$$


while in the ferromagnetic case they read as

$$
\begin{aligned}
c_{n, s} \Pi_{++}^{(r)++}(\mathbf{k}, \omega) & \approx \frac{\left(\lambda \xi^{\prime}\right)}{\left(\xi_{n, s}^{B}\right)^{2}} \frac{1}{k \lambda} \frac{i}{2} \ln \frac{\Omega-1}{\Omega+1}, \\
c_{n, s} \Pi_{00}^{(r) 00}(\mathbf{k}, \omega) & \approx \frac{\left(\lambda \xi^{\prime}\right)}{\left(\xi_{n, s}^{B}\right)^{2}} \frac{1}{k \lambda} \frac{i}{2} \ln \frac{\Omega-1+2 i \frac{\sqrt{\tilde{\gamma}}}{k \lambda}}{\Omega+1+2 i \frac{\sqrt{\tilde{\gamma}}}{k \lambda}} \\
c_{n, s} \Pi_{--}^{(r)--}(\mathbf{k}, \omega) & \approx \frac{\left(\lambda \xi^{\prime}\right)}{\left(\xi_{n, s}^{B}\right)^{2}} \frac{1}{k \lambda} \frac{i}{2} \ln \frac{\Omega-1+2 i \frac{\sqrt{2 \tilde{\gamma}}}{k \lambda}}{\Omega+1+2 i \frac{\sqrt{2 \tilde{\gamma}}}{k \lambda}} .
\end{aligned}
$$

These results can be obtained with the Mittag-Leffler expansion of their spectral functions similarly as for the scalar case [31]. The approximation of the spin wave polarization functions can be obtained in a similar way, but due to their different symmetry properties compared with density polarization functions another limit is to be taken in the spectral function. The calculation is outlined in the appendix. The results are

$$
\left|c_{s}\right|\left(\Pi_{+0}^{(r) 0+}+\Pi_{0-}^{(r)-0}\right)=\frac{C_{-}-C_{+}}{\widetilde{\gamma}}+\left[\frac{2 C_{0}+C_{+}+C_{-}}{\widetilde{\gamma}^{2}}+\frac{\Omega}{\widetilde{\gamma}^{2}}\left(C_{-}-C_{+}\right)+\frac{D_{-}-D_{+}}{\widetilde{\gamma}^{3}}\right](k \lambda)^{2}+\mathcal{O}\left((k \lambda)^{4}\right),
$$

with

$$
\begin{aligned}
C_{0} & =\frac{\lambda \xi^{\prime}}{\left(\xi_{s}^{B}\right)^{2}} \frac{2 \Gamma\left(\frac{3}{2}\right) F\left(\frac{3}{2} \mid \widetilde{\gamma}\right)}{\pi}, \\
C_{+} & =\frac{\lambda \xi^{\prime}}{\left(\xi_{s}^{B}\right)^{2}} \frac{\Gamma\left(\frac{3}{2}\right) F\left(\frac{3}{2} \mid 0\right)}{\pi}, \\
C_{-} & =\frac{\lambda \xi^{\prime}}{\left(\xi_{s}^{B}\right)^{2}} \frac{\Gamma\left(\frac{3}{2}\right) F\left(\frac{3}{2} \mid 2 \widetilde{\gamma}\right)}{\pi}, \\
D_{+} & =\frac{\lambda \xi^{\prime}}{\left(\xi_{s}^{B}\right)^{2}} \frac{4 \Gamma\left(\frac{5}{2}\right) F\left(\frac{5}{2} \mid 0\right)}{3 \pi} \\
D_{-} & =\frac{\lambda \xi^{\prime}}{\left(\xi_{s}^{B}\right)^{2}} \frac{4 \Gamma\left(\frac{5}{2}\right) F\left(\frac{5}{2} \mid 2 \widetilde{\gamma}\right)}{3 \pi}
\end{aligned}
$$

and $\widetilde{\gamma}$ is given by (137)).

In the case of linear dispersion $\Omega \gg 1$ can be assumed (while still satisfying the $|\Omega k \lambda| \ll 1$ condition). For the ferromagnetic state we further restrict ourselves to $\sqrt{\tilde{\gamma}} \ll|\Omega k \lambda|$. We will see that this later condition is equivalent with $c_{n} /\left|c_{s}\right| \gg 1$, which is fulfilled in the applications. In this case the regular polarization functions with zero spin transfer (138) can be further approximated by

$$
\left|c_{n, s}\right| \Pi^{(r)}(\mathbf{k}, \omega) \approx-\frac{\left(\lambda \xi^{\prime}\right)}{\left(\xi_{n, s}^{B}\right)^{2}} \frac{1}{k \lambda} \frac{i}{\Omega}
$$

both for the polar and ferromagnetic states. Since we are interested only in the low momentum behaviour of the dispersion curve $\Omega$ will be searched as a power series of the $(k \lambda)$ variable.

Polar case, density mode $(n=0)$ : The spectrum of these types of excitations can be determined by the poles of (116a). The corresponding equation is

$$
1-3 c_{n} \Pi_{0}-c_{n} \Pi_{S}=0 .
$$

Using Eqs. (137a ) and (140) and substituting $\Omega=a_{-1}(k \lambda)^{-1}+\mathcal{O}\left((k \lambda)^{0}\right)$, the resulting equation for the $a_{-1}$ coefficient reads as:

$$
a_{-1}^{2}+3 i \frac{\lambda \xi^{\prime}}{\left(\xi_{n}^{B}\right)^{2}} a_{-1}-\left(\frac{\lambda}{\xi_{n}^{B}}\right)^{2}=0 .
$$

Solving this quadratic equation (and assuming, that the imaginary part is small) one arrives at

$$
\Omega= \pm \frac{1}{k \xi_{n}^{B}}-i \frac{3}{2} \frac{\xi^{\prime}}{k\left(\xi_{n}^{B}\right)^{2}} .
$$


Returning to the more familiar variables one obtains

$$
\omega= \pm \sqrt{\frac{N_{0} c_{n}}{M}} k-i \frac{3 c_{n} M}{4 \pi \hbar^{3} \beta} k
$$

for the beginning of the dispersion curve.

Polar case, spin density mode $(n=0)$ : The excitation spectrum is obtained as the poles of (116b). The corresponding equation is

$$
1-2 c_{s} \Pi_{0}=0 .
$$

With the use of Eq. (140) the resulting equation for $\Omega$ reads as:

$$
1+2 i \frac{\xi^{\prime}}{\left(\xi_{s}^{B}\right)^{2}} \frac{1}{\Omega k}=0 .
$$

The solution is at

$$
\Omega=-i \frac{2 \xi^{\prime}}{k\left(\xi_{s}^{B}\right)^{2}}
$$

or equivalently at

$$
\omega=-i \frac{c_{s} M}{\pi \hbar^{3} \beta} k
$$

for the beginning of the dispersion curve. Note that such a mode did not appear in the Bogoliubov approximation, because there $D_{z z}$ is identically zero.

Polar case, spin wave mode $(n= \pm 1)$ : The poles of the autocorrelation function $(116 \mathrm{~d})$ determine the spectrum of these types of excitations. The corresponding equation is

$$
1-2 c_{s} \Pi_{0}-c_{s} \Pi_{S}=0 .
$$

Using Eqs. (137a) and (140) and substituting $\Omega=a_{-1}(k \lambda)^{-1}+\mathcal{O}\left((k \lambda)^{0}\right)$, the resulting equation for the $a_{-1}$ coefficient in this case reads as

$$
a_{-1}^{2}+2 i \frac{\lambda \xi^{\prime}}{\left(\xi_{s}^{B}\right)^{2}} a_{-1}-\left(\frac{\lambda}{\xi_{s}^{B}}\right)^{2}=0
$$

Whence

$$
\Omega= \pm \frac{1}{k \xi_{s}^{B}}-i \frac{\xi^{\prime}}{k\left(\xi_{s}^{B}\right)^{2}}
$$

or equivalently

$$
\omega= \pm \sqrt{\frac{N_{0} c_{s}}{M}} k-i \frac{c_{s} M}{2 \pi \hbar^{3} \beta} k .
$$

Ferromagnetic case, density, spin density mode $(n=0)$ : The poles of the autocorrelation functions (122a) or (122b) or (122c) determine the spectrum of these excitations, which is given by the equation

$$
\left(1-c_{n} \Pi_{n n}\right)\left(1-c_{s} \Pi_{z z}\right)-c_{n} c_{s} \Pi_{n z}^{2}=0 .
$$

Substituting the approximations (137b) and (140) and $\Omega=a_{-1}(k \lambda)^{-1}+\mathcal{O}\left((k \lambda)^{0}\right)$, the resulting equation for the $a_{-1}$ coefficient reads as

$$
\alpha_{n} \alpha_{s} a_{-1}^{3}+i \alpha\left(3 \alpha_{s}-2 \alpha_{n}\right) a_{-1}^{2}-\left(\alpha_{s}-\alpha_{n}-6 \alpha^{2}\right) a_{-1}+i 5 \alpha=0,
$$

with $\alpha=\xi^{\prime} / \lambda, \alpha_{n, s}=\left(\xi_{n, s}^{B} / \lambda\right)^{2}$. In the discussed temperature region $\alpha_{s} \gg \alpha_{n} \gg \alpha^{2}$. The solution of the equation can be calculated perturbatively, with results:

$$
\Omega= \pm \sqrt{\frac{\alpha_{s}-\alpha_{n}}{\alpha_{n} \alpha_{s}}} \frac{1}{k \lambda}-i \frac{\alpha}{2} \frac{3 \alpha_{s}^{2}+2 \alpha_{n}^{2}}{\alpha_{n} \alpha_{s}\left(\alpha_{s}-\alpha_{n}\right)} \frac{1}{k \lambda}
$$


from which the frequency

$$
\omega= \pm \sqrt{\frac{N_{0}\left(c_{n}+c_{s}\right)}{M}} k-i \frac{M}{4 \pi \hbar^{3} \beta} \frac{3 c_{n}^{2}+2 c_{s}^{2}}{c_{n}+c_{s}} k
$$

We can see now, that the $\sqrt{\widetilde{\gamma}} \ll|\Omega k \lambda|$ condition is equivalent with the $1 \ll 2 c_{n} /\left|c_{s}\right|$ condition, which is well satisfied.

Ferromagnetic case, spin wave mode with $n= \pm 1$ : The excitation spectrum of the ferromagnetic spin waves is obtained from the poles of the autocorrelation function $(122 \mathrm{~d})$. The equation to be solved is

$$
1-\frac{c_{s}}{2}\left[\Pi_{+0}^{(r) 0+}+\Pi_{0-}^{(r)-0}+\Pi_{+0}^{(s) 0+}\right]=0
$$

Substituting the approximations Eq. (137d) and Eq. (139a) and that $\Omega=a_{0}+\mathcal{O}\left((k \lambda)^{2}\right)$ leads to

$$
1-\frac{\gamma_{0}}{\widetilde{\gamma}}+\frac{C_{-}-C_{+}}{\widetilde{\gamma}}+\frac{(k \lambda)^{2}}{\widetilde{\gamma}^{2}}\left[\left(1-a_{0}\right) \gamma_{0}+2 C_{0}+C_{+}+C_{-}+\frac{a_{0}}{\widetilde{\gamma}}\left(C_{-}-C_{+}\right)+\frac{D_{-}-D_{+}}{\widetilde{\gamma}}\right]+\mathcal{O}\left((k \lambda)^{4}\right)=0
$$

The equation for $\mathcal{O}\left((k \lambda)^{0}\right)$ is an identity, since $\widetilde{\gamma}=\gamma_{0}+\beta\left|c_{s}\right|\left(H^{++}-H^{--}\right)$. The $a_{0}$ constant can be determined from $\mathcal{O}\left((k \lambda)^{2}\right)$ order terms

$$
a_{0}=\frac{\widetilde{\gamma}\left(2 C_{0}+C_{+}+C_{-}+\gamma_{0}\right)-D_{+}+D_{-}}{\widetilde{\gamma}^{2}}
$$

The frequency of the spin wave excitation then is a quadratic one

$$
\omega=\frac{\hbar k^{2}}{2 M^{*}},
$$

with the effective mass

$$
M^{*}=\frac{M}{a_{0}} .
$$

The Bogoliubov solution $(106 \mathrm{~b})$ can be regained in the $T \rightarrow 0$ limit, since $C_{0}, C_{+}, C_{-}, D_{+}, D_{-} \propto T, \widetilde{\gamma}$ and $\gamma_{0}$ tend to $N_{0}(T=0)\left|c_{s}\right| / k_{B} T$.

Ferromagnetic case, quadrupolar spin wave mode: Since the autocorrelation function (122e) is proper, so the spectrum of the quadrupolar spin waves is determined by the poles of the polarization function $\Pi_{+-}^{-+}$. The singular polarization (119) has its pole at

$$
\Omega=1+\frac{2 \widetilde{\gamma}}{(k \lambda)^{2}}
$$

The regular polarization function $\Pi_{+-}^{(r)-+}$ has a logarithmic singularity in the same place. The corresponding excitational frequency is

$$
\omega=\frac{2 \mathcal{M}\left|c_{s}\right|}{\hbar}+\frac{\hbar k^{2}}{2 M} .
$$

Some final remark is appropriate about the validity of the calculation of these last two modes. The approximation (139a) is valid if $(k \lambda)^{2} / \widetilde{\gamma} \ll 1$. This is equivalent with the $k \ll \sqrt{4 M \mathcal{M}\left|c_{s}\right|} / \hbar$ condition, meaning that the approximation for the bubble breaks down near the transition temperature, where the magnetisation vanishes.

\section{CONCLUSIONS}

In the high temperature phase density and spin fluctuations do not couple to each other and to the Green's functions. The latter appear only in the intermediate states in the perturbational expansion for the correlation functions describing density and spin fluctuations. Correspondingly one can experience independent excitation branches of one-particle and collective type. As discussed in the present paper in detail collective and one-particle excitations can hybridize in the Bose-Einstein condensed phase due to the symmetry breaking, which is different in the polar and 
in the ferromagnetic phase resulting in different couplings. In the polar phase, however such hybridization does not occur for spin modes characterized by spin transfers zero and \pm 2 . The general results have been demonstrated in the RPA scheme. By treating the regular polarization contributions as perturbations, we obtained damping for a number of modes determined first in the Bogoliubov approximation 14, 15. In the ferromagnetic phase for the transverse spin mode, whose energy has been shown to agree with the free particle kinetic energy in the Bogoliubov approximation, it is found that the eigenfrequency remains proportional to $k^{2}$ in RPA, but with an effective mass, which approaches the mass of the atoms in the zero temperature limit. Moreover the gap in the quadrupolar spin mode gets a temperature dependent correction, namely it is proportional to the magnetization which tends to the condensate density in the zero temperature limit and then the gap coincides with that of the Bogoliubov approximation. These transverse and quadrupolar spin modes are found free from damping.

There are thermal excitations for which the bubble graphs can not be treated as perturbations as shown earlier in the scalar case [31,39]. In the case of a spinor condensate one expects even a multitude of such excitations, already in RPA. Their investigation will be the subject of a forthcoming paper.

The calculations in this paper has been made for a homogeneous system. In experiments with alkali atoms the gas sample is confined in an optical trap which can be modelled by a harmonic potential. The inhomogeneous nature of the trapped system results in that the algebraic equations presented here are to be changed to coupled integral equations but their main structure remains the same. We note also that experiments can be designed where the local speed of sound can be measured directly making the results obtained for a homogeneous system also experimentally relevant.

\section{ACKNOWLEDGEMENTS}

The present work has been partially supported by the Hungarian Research National Foundation under grant No. OTKA T029552.

\section{APPENDIX A: THE APPROXIMATIONS OF THE CONTRIBUTIONS OF THE BUBBLE GRAPHS}

In this appendix we briefly outline the analytical properties of the contribution of the bubble graphs encountered and outline the approximation (139a). Some of these results are known from earlier works [31].

In the random phase approximation the self-energies of the internal lines are wavenumber and frequency independent, therefore the contribution of the bubble graph (109), depicted in Fig. 17 can be cast to the form

$$
\Pi_{r^{\prime} s^{\prime}}^{(r) s r}\left(\mathbf{k}, i \omega_{n}\right)=-\frac{1}{\hbar} \int \frac{d^{3} q}{(2 \pi)^{3}} \frac{n^{0}\left(\epsilon_{\mathbf{k}+\mathbf{q}}+\hbar \widetilde{\Sigma}^{s^{\prime} s}\right)-n^{0}\left(\epsilon_{\mathbf{q}}+\hbar \widetilde{\Sigma}^{r r^{\prime}}\right)}{i \omega_{n}-\hbar^{-1}\left(\epsilon_{\mathbf{k}+\mathbf{q}}-\epsilon_{\mathbf{q}}\right)-\Delta \widetilde{\Sigma}}
$$

with

$$
\Delta \widetilde{\Sigma}=\widetilde{\Sigma}^{s^{\prime} s}-\widetilde{\Sigma}^{r r^{\prime}}
$$

The nondiagonal elements of $\Pi_{r^{\prime} s^{\prime}}^{(r) s r}$ are zero, as stated below Eq. (109), the same is true for the proper self-energies. In the appendix the automatic summation over repeated indices do not apply.

Changing to retarded polarization functions with analytically continuing in frequency, the imaginary part plays the role of the spectral function:

$$
\Pi_{r s}^{(r) s r}(\mathbf{k}, \omega)=-\frac{1}{\pi} \int \frac{\mathfrak{I m} \Pi_{r s}^{(r) s r}\left(\mathbf{k}, \omega^{\prime}\right)}{\omega-\omega^{\prime}} d \omega^{\prime}
$$

The imaginary part can be brought to the form

$$
\mathfrak{I m}_{r s}^{(r) s r}(\mathbf{k}, \omega)=\frac{m^{2}}{4 \pi \hbar^{4} k \beta}\left[\int_{\infty}^{y_{0}} d z \frac{2 z}{e^{z^{2}+\gamma_{b}}-1}-\int_{\infty}^{y_{1}} d z \frac{2 z}{e^{z^{2}+\gamma_{f}}-1}\right],
$$


with

$$
\begin{aligned}
y_{0} & =\frac{k \lambda}{2}\left(\Omega-1-\frac{\Delta \gamma}{(k \lambda)^{2}}\right), \\
y_{1} & =\frac{k \lambda}{2}\left(\Omega+1-\frac{\Delta \gamma}{(k \lambda)^{2}}\right), \\
\gamma_{f} & =\hbar \beta \widetilde{\Sigma}^{s s} \\
\gamma_{b} & =\hbar \beta \widetilde{\Sigma}^{r r} \\
\Delta \gamma & =\gamma_{f}-\gamma_{b}=\hbar \beta \Delta \widetilde{\Sigma}
\end{aligned}
$$

and $\Omega$ defined in Eq. (136). Introducing the function

$$
R_{\gamma}(z)=\int_{-\infty}^{\infty} \frac{2 x}{e^{x^{2}+\gamma}-1} \frac{1}{x-z} d x, \quad \text { for } \mathfrak{I m} z>0,
$$

which is to be continued to the whole complex plane, the function $\Pi_{r s}^{(r) s r}(\mathbf{k}, \omega)$ can be represented as

$$
\Pi_{r s}^{(r) s r}(\mathbf{k}, \omega)=\frac{m^{2}}{(4 \pi)^{2} \hbar^{4} k \beta}\left[\int_{\infty}^{y_{0}} R_{\gamma_{b}}(z) d z-\int_{\infty}^{y_{1}} R_{\gamma_{f}}(z) d z\right], \quad \text { for } \mathfrak{I m} \omega>0
$$

The analytic structure of $R_{\gamma}(z)$ is important for the approximations, it can be found in Ref. 31. If the self-energies of the forward and backward propagating one-particle lines are equal, i.e. in the polar case and for the $n=0$ mode in the ferromagnetic case, $\Delta \gamma=0$ and the expression (A11) reduces that of Ref. [31. If the conditions above Eq. (138) are fulfilled, the leading contribution of the Mittag-Leffler representation of $R_{\gamma}(z)$, reading as

$$
R_{\gamma}(z)=2 \sqrt{\pi} \zeta\left(\frac{1}{2}\right)-i \pi z-\frac{2 \pi}{\sqrt{\gamma}} \frac{z}{z+i \sqrt{\gamma}}+4 \pi i \sum_{n=1}^{\infty} \frac{1}{a_{n}^{2}-b_{n}^{2}}\left[\frac{a_{n}^{2}+\gamma}{z+a_{n}}-\frac{b_{n}^{2}+\gamma}{z+b_{n}}+\left(a_{n}-b_{n}\right)\left(\frac{\gamma}{a_{n} b_{n}}-1\right)\right],
$$

with

$$
\begin{array}{ll}
a_{n}=i r_{n} e^{i \frac{\varphi_{n}}{2}}, & b_{n}=i r_{n} e^{-i \frac{\varphi_{n}}{2}}, \\
r_{n}=\sqrt[4]{\gamma^{2}+4 n^{2} \pi^{2}}, & \varphi_{n}=\arctan \frac{2 n \pi}{\gamma},
\end{array}
$$

can then be used to arrive at the approximating formulas Eqs. (138), see Ref. [31].

If the self-energies of the forward and backward propagating one-particle lines of the bubble graph are different, the upper limits of the integral (A11) will diverge when $k$ goes to zero, see Eqs. (A5) and (A6). If one is interested in the long wavelength dynamics the asymptotic series of $R_{\gamma}(z)$ can then be used, namely

$$
R_{\gamma}(z) \sim-\frac{2}{z^{2}} \sum_{n} \frac{\Gamma\left(n+\frac{3}{2}\right) F\left(n+\frac{3}{2} \mid \gamma\right)}{z^{2 n}},
$$

where $\Gamma(s)$ is the Gamma function and $F(s \mid \gamma)$ is the Bose-Einstein integral (127) [31]. With the help of Eq. (A11) and Eq. (A15), the approximation (139a) can be obtained.

[1] D. Stamper-Kurn and W. Ketterle, in Les Houches, Session LXXII, Coherent atomic matter waves, edited by R. Kaiser, C. Westbrook, and F. David (EDP Sciences; Springer-Verlag, Les Ulis; Berlin, 2001), p. 137.

[2] D. M. Stamper-Kurn et al., Phys. Rev. Lett. 80, 2027 (1998).

[3] J. Stenger et al., J. Low Temp. Phys. 113, 167 (1998).

[4] J. Stenger et al., Nature 396, 345 (1999).

[5] H.-J. Miesner et al., Phys. Rev. Lett. 82, 2228 (1999).

[6] W.-J. Huang and S.-C. Gou, Phys. Rev. A 59, 4608 (1999). 
[7] C.K.Law, H. Pu, and N. Bigelow, Phys. Rev. Lett 81, 5257 (1998).

[8] T.-L. Ho and S. K. Yip, Phys. Rev. Lett. 84, 4031 (2000).

[9] Y. Castin and C. Herzog, e-print cond-mat/0012040.

[10] T. Isoshima, K. Machida, and T. Ohmi, Phys. Rev. A 60, 4857 (1999).

[11] W.-J. Huang and S.-C. Gou, e-print cond-mat/9905435.

[12] S.-K. Yip, Phys. Rev. Lett. 83, 4677 (1999).

[13] H. T. C. Stoof, E. Vliegen, and U. A. Khawaja, e-print cond-mat/0103194.

[14] T.-L. Ho, Phys. Rev. Lett. 81, 742 (1998).

[15] T. Ohmi and K. Machida, J. Phys. Soc. Jpn. 67, 1822 (1998).

[16] J.-P. Martikainen and K.-A. Suominen, e-print cond-mat/0106013.

[17] F. Zhou, e-print cond-mat/0106133.

[18] M. Koashi and M. Ueda, Phys. Rev. Lett. 84, 1066 (2000).

[19] E. Goldstein and P. Meystre, Phys. Rev. A 61, 1509 (1999).

[20] H. Pu et al., Phys. Rev. A 60, 1463 (1999).

[21] H. Pu, S. Raghavan, and N. P. Bigelow, Phys. Rev. A 61, 023602 (2000).

[22] C. V. Ciobanu, S.-K. Yip, and T.-L. Ho, Phys. Rev. A 61, 033607 (2000).

[23] T.-L. Ho and L. Yin, Phys. Rev. Lett. 84, 2302 (2000).

[24] M. Ueda, Phys. Rev. A 63, 013601 (2001).

[25] F. Zhou, e-print cond-mat/0102372.

[26] N. Robins, W. Zhang, E. A. Ostrovskaya, and Y. S. Kivshar, e-print cond-mat/0102372.

[27] F. K. Abdullaev, B. B. Baizakov, and V. V. Konotop, e-print cond-mat/0105565.

[28] M. H. Anderson et al., Science 269, 198 (1995).

[29] K. B. Davis et al., Phys. Rev. Lett. 75, 3969 (1995).

[30] A. Griffin, Excitations in a Bose-condensed liquid (Cambridge University Press, Cambridge, 1993).

[31] P. Szépfalusy and I. Kondor, Ann. Phys. 82, 1 (1974).

[32] S.-K. Ma and C.-W. Woo, Phys. Rev. 159, 165 (1967).

[33] I. Kondor and P. Szépfalusy, Acta Phys. Hung. 24, 81 (1968).

[34] S. Payne and A. Griffin, Phys. Rev. B. 32, 7199 (1985).

[35] V. Wong and H. Gould, Ann. of Phys. 83, 252 (1974).

[36] G. Bene and P. Szépfalusy, Phys. Rev. A 58, R3391 (1998).

[37] J. Reidl, A. Csordás, R. Graham, and P. Szépfalusy, Phys. Rev. A 61, 043606 (2000).

[38] J. Reidl, G. Bene, R. Graham, and P. Szépfalusy, Phys. Rev. A 63, 043605 (2001).

[39] M. Fliesser, J. Reidl, P. Szépfalusy, and R. Graham, Phys. Rev. A 63, 043605 (2001).

[40] A. Fetter and J. Walecka, Quantum Theory of Many-Particle Systems (McGrow-Hill, New York, 1971).

[41] P. Szépfalusy and G. Szirmai, Phys. Rev. A 61, 051604(R) (2000).

[42] H. Shi and A. Griffin, Phys. Rep. 304, 1 (1998).

[43] P. Noziéres and D. Pines, The Theory of Quantum Liquids, Vol. I (Benjamin, New York, 1966). 